\title{
Discrete Approaches to Quantum Gravity in Four Dimensions
}

\author{
Renate Loll \\ Max-Planck-Institut für Gravitationsphysik \\ Schlaatzweg 1 \\ D-14473 Potsdam \\ e-mail: loll@aei-potsdam.mpg.de \\ Published 15 Dec 1998 \\ www.livingreviews.org/Articles/Volume1/1998-13loll \\ Living Reviews in Relativity \\ Published by the Max-Planck-Institute for Gravitational Physics \\ Albert Einstein Institute, Potsdam, Germany
}

\begin{abstract}
The construction of a consistent theory of quantum gravity is a problem in theoretical physics that has so far defied all attempts at resolution. One ansatz to try to obtain a non-trivial quantum theory proceeds via a discretization of space-time and the Einstein action. I review here three major areas of research: gauge-theoretic approaches, both in a path-integral and a Hamiltonian formulation; quantum Regge calculus; and the method of dynamical triangulations, confining attention to work that is strictly four-dimensional, strictly discrete, and strictly quantum in nature.
\end{abstract}

(c)1998 Max-Planck-Gesellschaft and the authors. Further information on copyright is given at http://www.livingreviews.org/Info/Copyright/. For permission to reproduce the article please contact livrev@aei-potsdam.mpg.de. 


\section{Article Amendments}

On author request a Living Reviews article can be amended to include errata and small additions to ensure that the most accurate and up-to-date information possible is provided. For detailed documentation of amendments, please go to the article's online version at

http://www. livingreviews .org/Articles/Volume1/1998-13loll/.

Owing to the fact that a Living Reviews article can evolve over time, we recommend to cite the article as follows:

\section{Loll, R.,}

"Discrete Approaches to Quantum Gravity in Four Dimensions", Living Rev. Relativity, 1, (1998), 13. [Online Article]: cited on <date>, http://www.livingreviews.org/Articles/Volume1/1998-13loll/.

The date in 'cited on $\langle$ date $>$ ' then uniquely identifies the version of the article you are referring to. 


\section{Contents}

1 Introduction $\quad 5$

2 Gauge-theoretic discretizations of gravity $\quad 6$

2.1 Lagrangian treatment. Introduction . . . . . . . . . . . . 6

2.2 Smolin's lattice model . . . . . . . . . . . . . . . 7

2.3 Numerical implementation . . . . . . . . . . . . . . . . . . . 7

2.4 Other gauge formulations . . . . . . . . . . . . . . . . . 7

2.5 Proving reflection positivity .............. 8

2.6 The measure .................... . . . . 8

2.7 Assorted topics . . . . . . . . . . . . . . . . . . 9

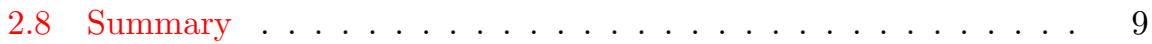

2.9 Hamiltonian treatment. Introduction . . . . . . . . . . . 9

2.10 Hamiltonian lattice gravity . . . . . . . . . . . . . . . . . . . . 10

2.11 The measure . . . . . . . . . . . . . . . . . . . . . . . . . . . . . 11

2.12 The constraint algebra . . . . . . . . . . . . . . . 11

2.13 Solutions to the Wheeler-DeWitt equation . . . . . . . . . . . 11

2.14 The role of diffeomorphisms . . . . . . . . . . . . . . . . . 12

2.15 The volume operator . . . . . . . . . . . . . . . . . . 12

2.16 The real dynamics . . . . . . . . . . . . . . . . . . . . . . . . . . . . . . . . . 13

2.17 Summary ............................. 14

3 Quantum Regge Calculus 15

3.1 Path integral for Regge calculus . . . . . . . . . . . . . 15

3.2 Higher-derivative terms . . . . . . . . . . . . . . . 16

3.3 First simulations . . . . . . . . . . . . . . . . 16

3.4 The phase structure ................... . . . . . . . . . . . . . . . . . 17

3.5 Influence of the measure . . . . . . . . . . . . . . . . 18

3.6 Evidence for a second-order transition? . . . . . . . . . . . . . . . 18

3.7 Avoiding collapse . . . . . . . . . . . . . . . . . 18

3.8 Two-point functions . . . . . . . . . . . . . . . . . . . . . . . . . 19

3.9 Non-hypercubic lattices . . . . . . . . . . . . . . . . . . . . . . . 19

3.10 Coupling to SU(2)-gauge fields . . . . . . . . . . . . . . . . 19

3.11 Coupling to scalar matter . . . . . . . . . . . . . . . 20

3.12 Recovering the Newtonian potential . . . . . . . . . . . . 20

3.13 Gauge invariance in Regge calculus? . . . . . . . . . . . . . . . . 21

3.14 Assorted topics . . . . . . . . . . . . . . . . . 21

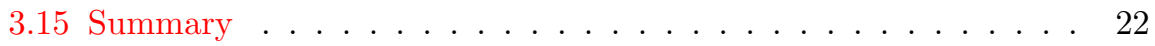

4 Dynamical triangulations 23

4.1 Introduction . . . . . . . . . . . . . . . . . . 23

4.2 Path integral for dynamical triangulations . . . . . . . . . . . . . . . . . . . . . . . . . . . . 23

4.3 Existence of an exponential bound?. . . . . . . . . . . . . . . 24

4.4 Performing the state sum ................... 24

4.5 The phase structure . . . . . . . . . . . . . . . 25 
4.6 Evidence for a second-order transition? . . . . . . . . . . . . . . 26

4.7 Influence of the measure . . . . . . . . . . . . . . . . . . 26

4.8 Higher-derivative terms . . . . . . . . . . . . . . . . . . 27

4.9 Coupling to matter fields . . . . . . . . . . . . . . . 27

4.10 Non-spherical lattices . . . . . . . . . . . . . . . . . . . . . . . . . . . . . . . . 28

4.11 Singular configurations . . . . . . . . . . . . . . . . . . . . . . . . . . . . . . 28

4.12 Renormalization group . . . . . . . . . . . . . . 28

4.13 Exploring geometric properties . . . . . . . . . . . . . . . 28

4.14 Two-point functions . . . . . . . . . . . . . . . . . . . 29

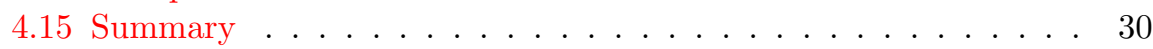

5 Conclusions and Outlook $\quad 31$

$\begin{array}{ll}\text { References } & 33\end{array}$

Living Reviews in Relativity (1998-13)

http://www.livingreviews.org 


\section{Introduction}

With the absence of a satisfactory quantum theory of gravity, a major puzzle in theoretical physics still remains unsolved. This article contains an overview and a comprehensive bibliography of past efforts to define a consistent theory of quantum gravity in four dimensions via an intermediate discretization. I will only discuss models with some concrete implementation of the dynamics of Einstein's theory, Lagrangian or Hamiltonian.

One way of tackling the quantization problem non-perturbatively is to use discrete methods, in analogy with quantum field theories on a flat background. They of course have to be modified in the case of gravity, where the metric of space-time becomes itself a dynamical variable. The diffeomorphism invariance of the classical continuum theory is no obstacle in principle to the introduction of a discretization, as has been demonstrated by the success of such methods in describing two-dimensional Euclidean gravity in a path-integral approach.

The approaches I will describe below have yielded a variety of interesting results on the discrete simulation methods themselves, as well as on the geometric properties of typical configurations, the role of gauge invariance, the phase structure, and the inclusion of matter, to name just a few. So far, they have not been successful in providing convincing evidence for the existence of a non-trivial four-dimensional quantum theory of gravity (neither, of course, have other methods). There are considerable technical difficulties in performing analytical computations and setting up sufficiently big numerical simulations. It is a theoretical challenge to come up with more realistic models that may lead to a truly interacting gravitational theory.

I have divided the discrete approaches into three categories, which I will discuss in the order of their chronological appearance: the gauge-theoretic formulations using connection variables, based on first-order descriptions of Einstein gravity and two metric formulations; the quantum Regge calculus program; and the more recent method of dynamical triangulations, which use simplicial instead of hypercubic lattice discretizations. They mainly deal with pure gravity, with the possible inclusion of a cosmological constant term and higher-order derivative terms, and in some instances, matter-coupling.

This review covers about 200 papers, the earliest of which appeared in 1979 (not counting the 1964 article by Leutwyler [145], who used a lattice approximation to perform a gravitational sum over histories à la Feynman). Much of the motivation for the discrete investigations comes of course from other sources, which for the most part I do not cite explicitly. The reader is referred to the individual articles for a more complete bibliography of the background material. 


\section{Gauge-theoretic discretizations of gravity}

\subsection{Lagrangian treatment. Introduction}

This area of research was inspired by the success of non-perturbative lattice methods in treating non-abelian gauge theories [173]. To apply some of their techniques, gravity has to be brought into a gauge-theoretic, first-order form, with the pure-gravity Lagrangian

$$
S[A, e]=\int_{M} e \wedge e \wedge R[A]
$$

where the $s o(3,1)$-valued spin-connection $A_{\mu}^{I J}$ (with curvature $R$ ) and the vierbein $e_{\mu}^{I}$ are considered as independent variables. The important feature of (1) is that $A_{\mu}^{I J}$ is a gauge potential, and that the action - in addition to its diffeomorphism-invariance - is invariant under local frame rotations. Variation with respect to $e_{\mu}^{I}$ leads to the metricity condition,

$$
\mathcal{D}_{[\mu} e_{\nu]}^{I} \equiv \partial_{[\mu} e_{\nu]}^{I}+A_{[\mu J}^{I} e_{\nu]}^{J}=0
$$

which can be solved to yield the unique torsion-free spin connection $A=A[e]$ compatible with the $e_{\mu I}$. There are some obvious differences with usual gauge theories: i) the action (1) is linear instead of quadratic in the curvature twoform $R_{\mu \nu}^{I J}$ of $A$, and ii) it contains additional fields $e_{\mu}^{I}$. Substituting the solution to (2) into the action, one obtains $S[e]=\int_{M} d^{4} x$ (det $\left.e\right) R$, where $R$ denotes the four-dimensional curvature scalar. This expression coincides with the usual Einstein action $\int d^{4} x \sqrt{\operatorname{det} g} R$ only for $\operatorname{det} e>0$.

Most of the lattice gauge formulations I will discuss below share some common features. The lattice geometry is hypercubic, defining a natural global coordinate system for labelling the lattice sites and edges. The gauge group $G$ is $S O(3,1)$ or its "Euclideanized" form $S O(4)$, or a larger group containing it as a subgroup or via a contraction limit. Local curvature terms are represented by (the traces of) $G$-valued Wilson holonomies $U_{\square}$ around lattice plaquettes. The vierbeins are either considered as additional fields or identified with part of the connection variables. The symmetry group of the lattice Lagrangian is a subgroup of the gauge group $G$, and does not contain any translation generators that appear when $G$ is the Poincaré group.

When discretizing conformal gravity (where $G=S O(5,1)$ ) or higher-derivative gravity in first-order form, the metricity condition on the connection has to be imposed by hand. This leads to technical complications in the evaluation of the functional integral.

The diffeomorphism invariance of the continuum theory is broken on the lattice; only the local gauge invariances can be preserved exactly. The reparametrization invariance re-emerges only at the linearized level, i.e. when considering small perturbations about flat space.

Living Reviews in Relativity (1998-13)

http://www . livingreviews .org 


\section{$2.2 \quad$ Smolin's lattice model}

The first gauge-theoretic model for lattice gravity is due to Smolin [186], based on the continuum formulation of MacDowell and Mansouri [157], with de Sitter gauge group $O(3,2)$ or $O(4,1)$, and a Lagrangian of $R^{2}$-type,

$$
S=\int d^{4} x \epsilon^{\mu \nu \rho \sigma} \epsilon_{I J K L} \tilde{R}_{\mu \nu}^{I J} \tilde{R}_{\rho \sigma}^{K L}
$$

where the components of $\tilde{R}_{\mu \nu}$ are related to those of the usual curvature tensor by

$$
\tilde{R}_{\mu \nu}^{I J}=R_{\mu \nu}^{I J} \pm \frac{1}{l^{2}}\left(e_{\mu}^{I} e_{\nu}^{J}-e_{\mu}^{J} e_{\nu}^{I}\right)
$$

Although the underlying gauge potentials $A_{\mu}$ are $o(3,2)$ - or $o(4,1)$-valued, the action is only invariant under the 6-dimensional subgroup of Lorentz transformations. The theory contains a dimensionful parameter $l$. The gauge potentials associated with the internal 5-direction are identified with the frame fields $e_{\mu}^{I}$, and the action can be decomposed into the usual Einstein-term (1) plus a cosmological constant term with $\lambda \sim \frac{1}{l^{2}}$ and a topological $R \wedge R$-term. Smolin analyzed its lattice discretization, and found both a weak- and a strong-coupling phase, with respect to the dimensionless coupling constant $g \sim \frac{\sqrt{G}}{l}$. He performed a weak-coupling expansion about flat space and rederived the usual propagator. In the strong-coupling regime he found massive excitations and a confining property for spinors.

\subsection{Numerical implementation}

Caracciolo and Pelissetto [63, 64, 65, 66, 67] performed a numerical investigation of the phase structure of Smolin's model. Using the compact group $S O(5)$ and its associated Haar measure, their findings confirmed the two-phase structure: a strong-coupling phase with a confining property and presence of exponential clustering, and a weak-coupling phase dominated by a class of topological configurations, with vanishing vierbein. However, their Monte Carlo data (on $4^{4}$ and $8^{4}$-lattices with periodic boundary conditions) indicated strongly that the transition was first-order, even if the measure was generalized by a factor of $|\operatorname{det} e|^{p}, p \in[0,150][67]$.

\subsection{Other gauge formulations}

Das et al [84] lattice-discretized an $S p(4)$-invariant Lagrangian due to West [194], leading to a functional form $S[U]=\sum_{n} \sqrt{\operatorname{Tr}\left(\left(U_{n}-\frac{1}{4} \operatorname{Tr} U_{n}\right)^{2}\right)}$, where $U_{n}$ denotes a sum of double-plaquette holonomies based at the vertex $n$. Details of the functional integration were not spelled out. The square-root form of the Lagrangian does not make it very amenable to numerical investigations (see also the comments in [139]).

Living Reviews in Relativity (1998-13)

http://www.livingreviews.org 
Mannion and Taylor [159] suggested a discretization of the $\left(\int e \wedge e \wedge R\right)$ Lagrangian without cosmological term, with the vierbeins $e$ treated as extra variables, and the compactified Lorentz group $S O(4)$.

Kaku [138] has proposed a lattice version of conformal gravity (see also $[137,139])$, based on the group $O(4,2)$, the main hope being that unitarity could be demonstrated non-perturbatively. As usual, a metricity constraint on the connection has to be imposed by hand.

A lattice formulation of higher-derivative gravity, containing fourth-order $R^{2}$-terms was given by Tomboulis [189]. The continuum theory is renormalizable and asymptotically free, but has problems with unitarity. The motivation for this work was again the hope of realizing unitarity in a lattice setting. The square-root form of the Lagrangian is similar to that of Das et al. It is $O(4)$-invariant and supposedly satisfies reflection positivity. Again, the form of the Lagrangian and the measure (containing a $\delta$-function of the notorsion constraint) is rather complicated and has not been used for a further non-perturbative analysis.

Kondo [142] employed the same framework as Mannion and Taylor, but introduced an explicit symmetrization of the Lagrangian. He claimed that the cluster expansion goes through just as in lattice Yang-Mills theory, leading to a positive mass gap.

\subsection{Proving reflection positivity}

Various properties of gauge gravity models were analyzed by Menotti and Pelissetto in a series of papers in the mid-eighties. They first studied a discrete $O(4)$-version of the Lagrangian (1) [165], including the $O(4)$-Haar measure and a general local $O(4)$-invariant measure for the vierbein fields, and showed that reflection-positivity holds only for a restricted class of functions. Furthermore, expanding about flat space, and after appropriate gauge-fixing, they discovered a doubling phenomenon similar to that found for chiral fermions in lattice gauge theory, a behaviour that also persists for different gauge-fixings. One finds the same mode doubling also for a flat-background expansion of conformal gravity [167]. In the same paper, they gave a unified treatment of Poincaré, de Sitter and conformal gravity, and showed that reflection positivity for $O(4)$-gravity (as well as for the two other gauge groups) holds exactly and for general functions only provided a signature factor $\operatorname{sign}(\operatorname{det} e)$ is included in the Lagrangian.

To ensure the convergence of the functional integration, one has to introduce a damping factor for the vierbeins in the measure, both for Poincare and conformal gravity [167]. An extension of the results of [167] to supergravity with the super-Poincaré group is also possible [62]. One can prove reflection positivity and finds a matching gravitino doubling in the perturbative expansion.

\subsection{The measure}

One can derive non-trivial conditions on the lattice measure by imposing the Slavnov-Taylor identities in a perturbative lattice calculation. Starting from the

Living Reviews in Relativity (1998-13)

http://www . livingreviews .org 
general form of the measure for Poincaré gravity,

$$
\prod_{n \mu \nu \rho \sigma} \operatorname{det}\left(e_{\mu}(n), e_{\nu}(n), e_{\rho}(n), e_{\sigma}(n)\right)^{N / 16} \prod_{n \mu} f\left(e_{\mu}^{2}(n)\right) \prod_{n, \mu>0} d e_{\mu}(n) \prod_{n, \mu>0} d U_{L}(n, n+\mu),
$$

Menotti and Pelissetto [166] performed a one-loop calculation and found that the Slavnov-Taylor identity can be satisfied for a particular choice of $N, f_{1}, f_{2}$ (momentum expansion coefficients of $f$ ), and of the cosmological constant $\lambda$. The solution still depends on a real parameter $\xi$ (related to a residual noninvariance under rotations). This makes it difficult to draw any immediate conclusions on the structure of the full, non-perturbative measure.

\section{7 $\quad$ Assorted topics}

Caselle et al [70] proposed a lattice action that is genuinely Poincaré-invariant, at the price of introducing additional lattice "coordinate variables". They also suggested a compact $O(5)$-formulation which reduces to the Poincare form in the limit as the length of some preferred $O(5)$-vector is taken to infinity, as well as a super-version involving the graded Poincaré group. The same authors in [69] put forward an argument for why doubling should appear in general gravity plus matter systems.

Reisenberger [175] has recently suggested a gauge-theoretic path integral based on the Plebanski action for Euclidean gravity. He discretizes the theory on a simplicial or hypercubic lattice with group- and algebra-valued fields. A metricity constraint needs to be imposed on the basic spin-1 fields, which it turns out is difficult to treat exactly.

\subsection{Summary}

The gauge-theoretic Lagrangian lattice approaches are afflicted by a number of technical difficulties. Reflection positivity can be shown for some of the models, but generally requires the inclusion of a factor $\operatorname{sign}(\operatorname{det} e)$ in the Lagrangian. Obtaining qualitative non-perturbative information about the phase structure requires a non-trivial measure input. The complicated functional form of the Lagrangian and the metricity condition that has to be imposed via a Lagrange multiplier and the corresponding functional integration do not make conformal and higher-derivative theories attractive candidates for numerical simulations. The compact version of Smolin's de Sitter gravity is still the simplest model, but its numerical investigation did not yield interesting results.

\subsection{Hamiltonian treatment. Introduction}

Relatively little work has been done on discretized Hamiltonian formulations of gravity. This can in part be understood from the fact that the numerical methods available for lattice gauge field theories rely mostly on the Euclidean path-integral description. Unfortunately the relation between the Lagrangian 
and Hamiltonian quantizations for generally covariant theories without a fixed background is far from clear.

In the usual metric formulation, the complicated non-polynomial form of the Hamiltonian constraint has been a long-standing problem. In this framework, neither the functional form of the quantum representations nor the nature of the quantization problems suggest that a discrete approach might yield any advantages. This situation has improved with the introduction of new Hamiltonian gauge-theoretic variables by Ashtekar [22, 23].

By a Hamiltonian lattice approach one usually means a formulation in which the time variable is left continuous, and only the spatial 3-slices are discretized. In continuum gravity, the $3+1$ decomposition leads to the (non-Lie) Dirac algebra of the three-dimensional diffeomorphism generators and the Hamiltonian constraint, associated with the deformation of three-surfaces imbedded in fourspace. One usually requires this algebra to be realized in the quantum theory, without anomalous terms, for a set of self-adjoint quantum constraint operators, for some factor-ordering.

Since a discretization of space-time breaks the diffeomorphism invariance, there is no reason to expect the Dirac algebra to be preserved in any discrete approach, even classically. This raises the question of whether and in what form part of the diffeomorphism symmetry can still be realized at the discrete level. Using gauge-theoretic variables, one can maintain the exact local gauge invariance with respect to the internal degrees of freedom, but there is no analogous procedure for treating the coordinate invariance.

\subsection{Hamiltonian lattice gravity}

There is a gauge-theoretic Hamiltonian version of gravity defined on a cubic lattice, which in many aspects resembles the Lagrangian gauge formulations described earlier. It also is virtually the only discrete Hamiltonian formulation in which some progress has been achieved in the quantization (see also [155] for a recent review).

Renteln and Smolin [180] were the first to set up a continuous-time lattice discretization along the lines of Hamiltonian lattice gauge theory. Their basic configuration variables are the link holonomies $U(l)$ of the spatial Ashtekar connection along the edges. The lattice analogues of the canonically conjugate pairs $\left(A_{a}^{i}(x), E_{i}^{a}(x)\right)$ are the link variables $\left(U(l)_{A}{ }^{B}, p_{i}(l)\right)$, with Poisson brackets

$$
\begin{aligned}
& \left\{U_{A}{ }^{B}(n, \hat{a}), U_{C}^{D}(m, \hat{b})\right\}=0, \\
& \left\{p_{i}(n, \hat{a}), U_{A}^{C}(m, \hat{b})\right\}=-\frac{1}{2} \delta_{n m} \delta_{\hat{a} \hat{b}} \tau_{i A}{ }^{B} U_{B}^{C}(n, \hat{a}), \\
& \left\{p_{i}(n, \hat{a}), p_{j}(m, \hat{b})\right\}=\delta_{n m} \delta_{\hat{a} \hat{b}} \epsilon_{i j k} p_{k}(n, \hat{a}),
\end{aligned}
$$

with the $S U(2)$-generators satisfying $\left[\tau_{i}, \tau_{j}\right]=2 \epsilon_{i j k} \tau_{k}$. Lattice links $l=(n, \hat{a})$ are labelled by a vertex $n$ and a lattice direction $\hat{a}$. These relations go over to the usual continuum brackets in the limit as the lattice spacing $a$ is taken to zero. In

Living Reviews in Relativity (1998-13)

http://www.livingreviews.org 
this scheme, they wrote down discrete analogues of the seven polynomial firstclass constraints, and also attempted to interpret the action of the discretized diffeomorphism and Hamiltonian constraints in terms of their geometric action on lattice Wilson loop states.

\subsection{The measure}

There is a natural measure for the quantum theory, given by the product over all lattice edges of the Haar measures $d g$. However, since the Ashtekar connections $A$ are complex-valued, the gauge group is the non-compact group $S O(3, \mathbb{C})=S L(2, \mathbb{C})$, and the gauge-invariant Wilson loop functions are not square-integrable. For the alternative formulation in terms of real $\mathrm{SU}(2)$-variables (see below), these problems are not present. An alternative heat kernel measure $d \nu$ for holomorphic $S L(2, \mathbb{C})$ holonomies on the lattice was used in [146, 99].

\subsection{The constraint algebra}

This line of research was continued by Renteln [179], who proved that for a particular factor-ordering (all momenta to the left), the subalgebra of the discretized diffeomorphism constraints (smeared by lapse functions $N$ ),

$$
\sum_{n} \mathcal{V}^{\text {latt }}[N, n):=\sum_{n} N^{\text {latt }}(n, \hat{a}) \operatorname{Tr}\left(U_{\square_{a b}} \tau_{i}\right) p_{i}(n, \hat{b}),
$$

closes in the limit as the lattice spacing is taken to zero. This calculation was later extended to a variety of different symmetrizations for the lattice operator and to an arbitrary factor-ordering of the form $\alpha \operatorname{Tr}(\hat{U} \tau) \hat{p}+(1-\alpha) \hat{p} \operatorname{Tr}(\hat{U} \tau)$, with $0 \leq \alpha \leq 1$ [156]. Again, one does not find any quantum anomalies. It would be highly desirable to extend this result to commutators involving also the discretized Hamiltonian constraint and to find the explicit functional form of the anomalies, if there were any.

\subsection{Solutions to the Wheeler-DeWitt equation}

Part of solving the canonical quantum theory is to determine the states annihilated by the Hamiltonian constraint $\hat{H}$. It was shown by Loll [146] that solutions exist in the Renteln-Smolin formulation, where $\hat{H}(n)=\sum_{a, b} \epsilon^{i j k} \operatorname{Tr}\left(\hat{U}\left(n, \square_{\hat{a} \hat{b}}\right) \tau_{k}\right) \hat{p}_{i}(n, a) \hat{p}_{j}(n, b)$. They are given by multiple, non-intersecting Polyakov loops (the lattice is assumed to have compact topology $T^{3}$ ). Such solutions are trivial in the sense that they correspond to quantum states "without volume". The difficulties one encounters when trying to find other solutions is illustrated by the explicit calculations for the $1 \times 1 \times 1$-lattice in [146].

The search for solutions was continued by Ezawa [99] (see also [100] for an extensive review), who used a symmetrized form of the Hamiltonian. His solutions depend on multiple plaquette loops $\left(U_{\square}\right)^{k}$, where a single lattice plaquette $\square$ is traversed by the loop $k$ times. The solutions are less trivial than those formed 
from Polyakov loops, since they involve kinks, but they are still annihilated by the volume operator.

A somewhat different strategy was followed by Fort et al [102], who constructed a Hamiltonian lattice regularization for the calculation of certain knot invariants. They defined lattice constraint operators in terms of their geometric action on lattice Wilson loop states, and reproduced some of the formal continuum solutions to the polynomial Hamiltonian constraint of complex Ashtekar gravity on simple loop geometries.

\subsection{The role of diffeomorphisms}

As in other discrete formulations, the spatial diffeomorphism group cannot be realized exactly on the lattice, and the only obvious symmetries of a cubic lattice are discrete rotations and overall translations. The commutator computation described above indicates that one may be able recover the diffeomorphism invariance in a suitable continuum limit. Corichi and Zapata [83] have suggested the presence of a residual diffeomorphism symmetry in the lattice theory, under which, for example, all non-intersecting Wilson loop lattice states would be identified.

One can try to interpret the lattice theory as a manifestly diffeomorphisminvariant construction, with the lattice representing an entire diffeomorphism equivalence class of lattices embedded in the continuum [146]. In order to make this interpretation consistent, one should modify the functional form of either the Hamiltonian or the measure, in such a way that the commutator of two lattice Hamiltonians vanishes, as $a \rightarrow 0$.

\subsection{The volume operator}

An important quantity in Hamiltonian lattice quantum gravity is the volume operator, the quantum analogue of the classical volume function $\int d^{3} x \sqrt{\operatorname{det} g}$. The continuum dreibein determinant $\operatorname{det} E(x)$ (with $|\operatorname{det} E(x)|=\operatorname{det} g$ ), has a natural lattice analogue, given by

$$
\operatorname{det} p(n):=\frac{1}{3 !} \epsilon_{a b c} \epsilon^{i j k} p_{i}(n, \hat{a}) p_{j}(n, \hat{b}) p_{k}(n, \hat{c}) .
$$

Apart from characterizing geometric properties of lattice quantum states, it is needed in the construction of the quantum Hamiltonian of the real connection approach.

Loll [147] showed that a lattice Wilson loop state has to have intersections of valence at least 4 in order not to be annihilated by the volume operator

$\sum_{n} \sqrt{\operatorname{det} \hat{p}(n)}$. This result is independent of the choice of gauge group $(S U(2)$ or $S L(2, \mathbb{C}))$. The volume operator has discrete eigenvalues, and part of its non-vanishing spectrum, for the simplest case of four-valent intersections, was first calculated by Loll [149]. These spectral calculations were confirmed by De Pietri and Rovelli [94], who derived a formula for matrix elements of the volume operator on intersections of general valence (as did Thiemann [188]).

Living Reviews in Relativity (1998-13)

http://www.livingreviews.org 
This still leaves questions about the spectrum itself unanswered, since the eigenspaces of $\operatorname{det} \hat{p}(n)$ grow rapidly, and diagonalization of the matrix representations becomes a technical problem. Nevertheless, one can achieve a better understanding of some general spectral properties of the lattice volume operator, using symmetry properties. It was observed in [149] that all non-vanishing eigenvalues of $\operatorname{det} \hat{p}(n)$ come in pairs of opposite sign. Loll subsequently proved that this is always the case [151]. A related observation concerns the need for imposing an operator condition det $p>0$ on physical states in non-perturbative quantum gravity [151], a condition which distinguishes its state space from that of a gauge theory already at a kinematical level.

The symmetry group of the cubic three-dimensional lattice is the discrete octagonal group $\mathcal{O}$, leaving the classical local volume function $\operatorname{det} p(n)$ invariant. Consequently, one can find a set of operators that commute among themselves and with the action of the volume operator, and simplify its spectral analysis by decomposing the Hilbert space into the corresponding irreducible representations [154]. This method is most powerful when applied to states which are themselves maximally symmetric under the action of $\mathcal{O}$, in which case it leads to a dramatic reduction of the dimension of the eigenspaces of $\operatorname{det} \hat{p}(n)$.

In addition to the volume operator, one may define geometric lattice operators measuring areas and lengths $[150,152]$. They are based on (non-unique) discretizations of the continuum spatial integrals of the square root of the determinant of the metric induced on subspaces of dimension 2 and 1. For the case of the length operator, operator-ordering problems arise in the quantization.

\subsection{The real dynamics}

To avoid problems with the non-compactness of the gauge group and the formulation of suitable "quantum reality conditions", Barbero [25] advocated to use a real $\mathrm{su}(2)$-connection formulation for Lorentzian continuum gravity. This can be achieved, at the price of having to deal with a more complicated Hamiltonian constraint. Loll $[148,153]$ translated the real connection formulation to the lattice and studied some of the differences that arise in comparison with the complex approach. Adding for generality a cosmological constant term, this leads to a lattice Hamiltonian

$$
H^{\text {latt }}=\sum_{n} N^{\text {latt }}(n)\left[\mathcal{H}_{\text {kin }}^{\text {latt }}+\mathcal{H}_{\text {pot }}^{\text {latt }}+\sqrt{\lambda} G \sqrt{\operatorname{det} p(n)}\right]
$$

where schematically $\mathcal{H}_{\text {kin }}^{\text {latt }}=(\operatorname{det} p)^{-1 / 2} \operatorname{Tr}(U \tau) p^{2}, \mathcal{H}_{\text {pot }}^{\text {latt }}=((\operatorname{Tr}(\tau U p \tau U)-$ $p)^{2} p^{6}(\operatorname{det} p)^{-5 / 2}$. This regularized Hamiltonian is well-defined on states with $\hat{\operatorname{det} p} \neq 0$, but its functional form is not simple. The negative powers of the determinant of the metric can be defined in terms of the spectral resolution of det $p$. The type of representation and regularization enables one to handle this non-polynomiality. 


\subsection{Summary}

Some progress has been achieved in Hamiltonian lattice gravity, using discrete analogues of the Ashtekar variables. The quantization program is still openended, and no physically non-trivial solutions to the Wheeler-DeWitt equation are known. The fact that one can study and evaluate geometric operators provides useful characterization of quantum states.

An analysis of the spectrum of the volume operator is crucial for handling the non-polynomial terms in the quantum Hamiltonian constraint. It will be necessary to find a suitable truncation or approximation to simplify further the spectral analysis of the Wheeler-DeWitt operator. A suitable quantum analogue of the continuum limit $a \rightarrow 0$ has not yet been established, and in this regard the Hamiltonian ansatz does not go beyond the results obtained in the Lagrangian formulations described earlier.

Why should one bother with a Hamiltonian quantization at all? Typical quantities one wants to study in a discrete path-integral approach to gravity are transition amplitudes between three-geometries on different spatial slices. This is not complete without a specification of the corresponding quantum states, which are in principle elements of Hilbert spaces of discrete three-geometries of the type described above.

Living Reviews in Relativity (1998-13)

http://www.livingreviews.org 


\section{Quantum Regge Calculus}

\subsection{Path integral for Regge calculus}

A path-integral quantization of $4 \mathrm{~d}$ Regge calculus was first considered in the early eighties [181, 104, 81]. This approach goes back to Regge [174], who proposed approximating Einstein's continuum theory by a simplicial discretization of the metric space-time manifold and the gravitational action. Its local building blocks are four-simplices $\sigma$. The metric tensor associated with each simplex is expressed as a function of the squared edge lengths $l^{2}$ of $\sigma$, which are the dynamical variables of this model. For introductory material on classical Regge calculus and simplicial manifolds, see [168, 187, 104, 124, 106, 197, 190, 8]; various quantum aspects are reviewed in [106, 107, 112, 110, 116, 133, 40, 160, 190, 198].

One may regard a Regge geometry as a special case of a continuum Riemannian manifold, a so-called piecewise flat manifold, with a flat metric in the interior of its 4-simplices $\sigma$, and singular curvature assignments to its two-simplices $b$ (the bones or hinges).

The Einstein action with cosmological term in the Regge approach is given by

$$
S^{\text {Regge }}\left[l_{i}^{2}\right]=\sum_{\text {bones } b} V_{b}\left(\lambda-k \frac{A_{b} \delta_{b}}{V_{b}}\right) \sim \int d^{4} x \sqrt{g}\left(\lambda-\frac{k}{2} R\right),
$$

where $k=\frac{1}{8 \pi G}, A_{b}$ is the area of a triangular bone, $\delta_{b}=2 \pi-\sum_{\sigma \supset b} \theta(\sigma, b)$ the deficit angle there, and $V_{b}$ a local four-volume element. $\theta(\sigma, b)$ is the angle between the two 3-simplices of $\sigma(\pi$ minus the angle between their inward normals) intersecting in $b$. Hartle and Sorkin [128] have generalized (12) to the case of manifolds with boundary. The boundary contribution to the action is given by

$$
\sum_{b \subset \text { boundary }} A_{b} \psi_{b} \sim \int d^{3} x \sqrt{g^{(3)}} K,
$$

where $\psi_{b}$ is the angle between the normals of the two three-simplices meeting at $b$. The Euclidean path integral on a finite simplicial complex of fixed connectivity takes the form

$$
Z(k, \lambda)=\int \mathcal{D} l e^{-S^{\text {Regge }}\left[l_{i}^{2}\right]}
$$

with $\int \mathcal{D} l$ representing the discrete analogue of the sum over all metrics. A crucial input in (14) is the choice of an appropriate measure $\mathcal{D} l$. In general, a cutoff is required for both short and long edge lengths to make the functional integral convergent [104, 81, 18]. One is interested in the behaviour of expectation values of local observables as the simplicial complex becomes large, and the existence of critical points and long-range correlations, in a scaling limit and as the cutoffs are removed.

A first implementation of these ideas was given by Roček and Williams [181, 183]. They obtained a simplicial lattice geometry by subdividing each unit 
cell of a hypercubic lattice into simplices. Their main result was to rederive the continuum free propagator (see also [101] for related results) in the limit of weak perturbations about flat space. This calculation can be repeated for Lorentzian signature [196].

Some non-perturbative aspects of the path integral were investigated in [183] (see also [182]). In this work, discrete analogues of space-time diffeomorphisms are defined as the local link length transformations which leave the action invariant, and go over to translations in the flat case. It is argued that an approximate invariance should exist in $4 \mathrm{~d}$. One may define analogues of local conformal transformations on a simplicial complex by multiplication with a positive scale factor at each vertex, but the global group property is incompatible with the existence of the generalized triangle inequalities.

\section{$3.2 \quad$ Higher-derivative terms}

Simplicial analogues of higher-derivative terms were introduced in $[117,119]$. In the continuum, with an appropriate choice of coupling constants, their inclusion makes the path integral less ill-behaved. The simplest higher-derivative term in Regge calculus is given by

$$
\sum_{b} \frac{A_{b}^{2} \delta_{b}^{2}}{V_{b}} \sim \int d^{4} x \sqrt{g} R_{\mu \nu \rho \sigma} R^{\mu \nu \rho \sigma},
$$

with $V_{b}$ denoting the local Voronoi four-volume at $b$. The fact that (15) should not be identified with $\int d^{4} x \sqrt{g} R^{2}$ is less surprising in light of the classical result [81, 82], that Regge's expression $A_{b} \delta_{b}$ for the scalar curvature (for $d>2$ ) converges to its continuum counterpart not pointwise, but only after integration, i.e. "in the sense of measures" (see also [103, 101], where similar convergence properties were studied by using an imbedding into a sufficiently large vector space $\mathbb{R}^{N}$ ).

More complicated higher-curvature terms can in principle be constructed, using a simplicial analogue of the Riemann tensor (see, for example, [174, 106, $119,55]$ ), but have up to now not been used in numerical simulations. A related proposal by Ambjørn et al [19] is to include terms in the action that depend on higher powers of the deficit angle $\delta_{b}$, as well as terms containing powers of the solid angle $\delta_{v}$ at a vertex. The introduction of local vierbeins and parallel transporters is also necessary if one considers fermion coupling [104, 176].

\subsection{First simulations}

The first numerical studies of the Regge action were undertaken by Berg [38, 39] and Hamber and Williams [118, 119]. Berg performed a Monte-Carlo simulation of the pure-curvature action for hypercubic $2^{4}$ - and $3^{4}$-lattices with simplicial subdivision (see [40] for a description of the method). He used the scale-invariant measure $\mathcal{D} l=\prod_{i} \frac{d l_{i}}{l_{i}}$. To avoid the divergence that results from rescaling the link lengths, he kept the total volume constant by performing an overall length

Living Reviews in Relativity (1998-13)

http://www.livingreviews.org 
rescaling of all links after each move. This amounts to fixing a typical length scale $l_{0}:=\left(v_{0}\right)^{\frac{1}{4}}$, where $v_{0}$ is the expectation value of the 4 -simplex volume.

For $k=0$, he found a negative average curvature $\langle R\rangle$, and some evidence for a canonical scaling behaviour of lengths, areas and volumes. For $k= \pm 0.3$, he obtained a negative (positive) average deficit angle $\langle\delta\rangle$ and a positive (negative) $\langle R\rangle$. A more detailed analysis for $0 \leq k \leq 0.1$ led Berg [39] to conclude that there exists a critical value $k^{c}$ (presumably a first-order transition [40]), below which $\langle R\rangle$ is convergent, whereas above it diverges. (Myers [169] has conjectured that it may be possible to perform a similar analysis for Monte-Carlo data for the Lorentzian action.)

By contrast, Hamber and Williams [118] simulated the higher-derivative action

$$
S\left[l_{i}^{2}\right]=\sum_{b}\left[\lambda V_{b}-k \delta_{b} A_{b}+a A_{b}^{2} \delta_{b}^{2} / V_{b}\right]
$$

on $2^{4}$ - and $4^{4}$-lattices, using a time-discretized form of the Langevin evolution equation (see also [106]). For technical reasons, one uses barycentric instead of Voronoi volumes. They employed the scale-invariant measure $\mathcal{D} l=\prod_{i} \frac{d l_{i}}{l_{i}} F_{\epsilon}(l)$, where $F_{\epsilon}$ enforces an ultra-violet cutoff $\epsilon$. They investigated the average curvature $\mathcal{R} \sim\langle R\rangle$ and squared curvature $\mathcal{R}^{2}$ (scaled by powers of $\left\langle l^{2}\right\rangle$ to make them dimensionless), as well as $\left\langle\delta_{b}^{2}\right\rangle$ and $\left\langle V_{b}\right\rangle /\left\langle l^{2}\right\rangle$. For $\lambda=k=a=0$, one finds a negative $\mathcal{R}$ and a large $\mathcal{R}^{2}$, indicating a rough geometry. For small $a$, one observes a sudden decrease in $\mathcal{R}^{2}$, as well as a jump from large positive to small negative values of $\mathcal{R}$ as $\lambda$ is increased. For large $a, \mathcal{R}$ is small and negative, and the geometry appears to be smooth. Like Berg, they advocated a fundamental-length scenario, where the dynamically determined average link length provides an effective UV-cutoff.

\subsection{The phase structure}

Further evidence for a transition between a region of rough and smooth geometry comes from Monte Carlo simulations by Hamber $[108,111]$ on $4^{4}$ - and $8^{4}$-lattices, this time with the lattice $\mathcal{D} l=\prod_{i} l_{i} d l_{i}$ (see also [114] for a summary of results, and [109] for more details on the method). There is a value $k_{c}$ at which the average curvature vanishes. For $k>k_{c}$, the curvature becomes large and the simplices degenerate into configurations with very small volumes. He performed a simultaneous fit for $k_{c}, A_{\mathcal{R}}$ and $\delta$ in the scaling relation

$$
\mathcal{R}(k) \stackrel{k \rightarrow k_{c}}{\sim} A_{\mathcal{R}}\left(k_{c}-k\right)^{\delta} .
$$

This leads to a scaling exponent $\delta \approx 0.60$, with only a weak dependence on $a$. There are even points with $a=0$ that lie in the well-defined, smooth phase. Hamber also investigated the curvature and volume susceptibilities $\chi_{\mathcal{R}}$ and $\chi_{V}$. At a continuous phase transition, $\chi_{\mathcal{R}}$ should diverge, reflecting long-range correlations of a massless graviton excitation. The data obtained are not incompatible with such a scenario, but the extrapolation to the transition point $k_{c}$ seems somewhat ambiguous. On the other hand, one does not expect $\chi_{V}$ to diverge at $k_{c}$, which is corroborated by the simulations.

Living Reviews in Relativity (1998-13)

http://www.livingreviews.org 


\subsection{Influence of the measure}

For the pure Einstein action, (12) with $\lambda=0$, some differences between the $M_{1}=d l / l$ - and the $M_{2}=l d l$-measure were investigated on a $4^{4}$-lattice by Beirl et al $[29,30]$. A constant-volume constraint was used for simulations with the $M_{1}$-measure, and a cutoff $l^{2}<$ const for $M_{2}$. A study of the $k$-dependence of bulk geometric quantities agreed with previous simulations, wherever applicable. For small $k$, both measures lead essentially to identical results. For the $M_{1}$ measure only, one finds that $\langle R\rangle /\langle V\rangle$ and $\langle A\rangle$ exhibit a jump at some $k_{0}$, due to the formation of spikes (isolated long link lengths, with the areas staying small). ¿From this, and the study of edge length distributions, one concludes that the DeWitt-type measure $M_{2}$ is generally better behaved.

\subsection{Evidence for a second-order transition?}

The same $l d l$-measure was used by Hamber in an extension of a previous simulation of the higher-derivative action (16) [113], for lattice sizes of up to $16^{4}$. At the transition point $\left(k=k_{c} \simeq 0.244, \lambda=1, a=0.005\right)$, the distributions of edge lengths, volumes and curvatures are smooth and Gaussian-like, and the average curvature vanishes. The location of $k_{c}(a)$ from fits $(17)$ to the average curvature coincide with those from [111], leading to $\delta \simeq 0.626$. The data at $a=0$ do not seem to match this interpretation. This leads to the tentative conclusion that only for sufficiently large $a$ the observed transition is of second order. It is in general "difficult to entirely exclude the presence of a weak firstorder transition, if it has a very small latent heat". For $a=0.005$, one finds some evidence for a decrease in the fractal dimension as $k$ grows.

\subsection{Avoiding collapse}

Starting from the observation that Regge configurations exist with $R_{\text {tot }} \rightarrow \infty$ and $V_{\text {tot }} \rightarrow 0$, Beirl et al [31, 32] investigated the influence of a cutoff $f$ on the fatness of a simplex $\sigma$, defined by

$$
\phi_{\sigma}:=\frac{V_{\sigma}^{2}}{\max _{l \in \sigma} l^{8}} \geq f>0 .
$$

Such a uniform shrinking of simplices is known to be necessary in order for piecewise flat manifolds to approach their continuum counterparts [82]. On the $4^{4}$-lattice, with the $l d l$-measure and $\lambda=0$, they determined $\mathcal{R}(k)$ for decreasing $f$. For small $k$, the choice of $f$ seems to have only little influence, but towards the transition point $k_{c},\left\langle\phi_{\sigma}\right\rangle$ simultaneously decreases. Next they investigated a variety of measures of the form $l^{2 \sigma-1} d l$ (see also [35]). For $\sigma \leq 1$, only a mild $\sigma$-dependence of $\mathcal{R}$ is observed as $k \rightarrow k_{c}$. However, for $\sigma=1.5$, there are significant differences for the entire range of $k$, and some evidence that the geometry degenerates.

Living Reviews in Relativity (1998-13)

http://www.livingreviews.org 


\subsection{Two-point functions}

To understand the nature of the possible excitations at the phase transition, one needs to study correlation functions in the vicinity of $k_{c}$, which is difficult numerically. Some data are available on the connected correlation functions of the curvatures and the volumes at fixed geodesic distance $d, G_{R}(d)$ and $G_{V}(d)$, for lattice sizes $\leq 16^{4}$ [115], using a scalar field propagator to determine $d$. Both correlators were measured at various $k$-values, leading to similar results for both $a=0$ and $a=0.005$. The data, taken for $d \leq 16$ ( $\simeq 7$ lattice spacings), can be fitted to decaying exponentials.

Some further data (for $a=0$ ) were reported by the Vienna group [37, 34]. These authors simply used the lattice distance $n$ instead of the true geodesic distance $d$. In [37], the measure was taken to be of the form $l^{2 \sigma-1} d l$. They looked at $G_{V}(n)$ on $3^{3} \times 8$ - and $4^{3} \times 16$-lattices, for $\lambda=\sigma=1$ and $\lambda=\sigma=0.1$, and found a fast decay for all investigated values of $k$, and $n \leq 8$.

\section{$3.9 \quad$ Non-hypercubic lattices}

Simulations on lattices with irregular link geometry (still with $T^{4}$-topology) have been performed by Beirl et al [31, 36]. They were obtained by adding a few vertices of low coordination number to otherwise regular lattices. One finds that the average curvature $\langle R\rangle$ increases from negative to positive values, even for $k<k_{c}$, as a result of the formation of spikes. In [36], the averages $\left\langle l^{2}\right\rangle$ were monitored separately at the regular and the inserted vertices.

The dependence of $\mathcal{R}$ on $k$ is rather interesting: One observes two "critical" points, a smaller one $k_{1}$, where the extra vertices develop spikes, and a second one $k_{2}$ where the remaining vertices follow. $\mathcal{R}$ undergoes a small jump at $k_{1}$, and a larger one at $k_{2}$. There is also a transition point to a phase with collapsed simplices at large negative $k$, with a jump to large negative $\mathcal{R}$. Additional transition points at negative $k$ were also found in simulations of the "compactified" Regge action $S=\sum_{b}\left(-k A_{b} \sin \delta_{b}+\lambda V_{b}\right)$ (this action was discussed in [104]; see also [71, 140]) and a $\mathbb{Z}_{2}$-version of Regge gravity [33]. Correlation functions at those points were computed in [34] for short distances, but no evidence for long-range correlations was found.

The same authors studied the inclusion of the higher-derivative term (15) in [36]. On the regular lattice, their findings for $\mathcal{R}(k, a)$ confirmed those by Hamber, apart from the fact that they found stable expectation values even for positive $\mathcal{R}$. Inserting irregular vertices pushes $\mathcal{R}$ to larger values and leads again to the appearance of an additional transition point.

\subsection{Coupling to SU(2)-gauge fields}

Berg and collaborators $[42,43,44,27,28,41]$ coupled the pure-curvature action geometrically to the Wilson action for $\mathrm{SU}(2)$-gauge fields via dimensionless 
weight factors $W_{b}$,

$$
S=S^{\text {Regge }}-\frac{\beta}{2} \sum_{b} W_{b} \operatorname{Re}\left[\operatorname{Tr}\left(1-U_{b}\right)\right], \quad W_{b}=\text { const } \frac{V_{b}}{\left(A_{b}\right)^{2}},
$$

where $U_{b}$ denotes the $\mathrm{SU}(2)$-holonomy around $b$ and $\beta$ is proportional to the inverse square coupling constant, $\beta=\frac{4}{g^{2}}$. One motivation was to understand whether in the well-defined pure-gravity region, one can choose the elementary particle masses to be $\ll m_{\mathrm{Pl}}$ as $\beta \rightarrow \infty$, as one might expect for a realistic gravity + matter system. This seems a rather distant hope, since in the simulations performed so far, the ratio $m_{\mathrm{Pl}} / m_{\text {hadron }}$ is of order unity.

Initial computations were performed on a $2 \times 4^{3}$-lattice with the scaleinvariant measure, and at $k=0.01$ [42, 44], and extended to larger $k$-values in [43]. For $k \leq 0.04$, one finds some evidence for a (first-order?) transition; the region of $\beta$ where the transition occurs does not change much with $k$. Beirl et al $[27,28]$ extended this analysis by measuring the static potential $V$ of a quark-antiquark pair on lattices of size $4 \times 6^{3}$ and $4 \times 8^{3}$. With and without gravity, one finds both a confined and a deconfined phase; in the presence of gravity, the transition occurs at a smaller $\beta$-value. More recently, Berg et al [41] have gathered further data on the location and stability of the well-defined phase in the $(k, \beta)$-plane, and extracted a string tension for various $\beta$-values.

\subsection{Coupling to scalar matter}

The coupling of Regge gravity to scalar matter was investigated by Hamber and Williams $[120,116]$ for $\lambda=1, a=0.005$, and various $k$-values. They considered a single, massive scalar field, with an action contribution

$$
S_{\phi}=\frac{1}{2} \sum_{<i j>} V_{i j}\left(\frac{\phi_{i}-\phi_{j}}{l_{i j}}\right)^{2}+\frac{m^{2}}{2} \sum_{i} V_{i} \phi_{i}^{2}
$$

where $V_{i}, V_{i j}$ denote the 4 -volumes associated with the vertices and edges. Most data were obtained on the $4^{4}$-lattice, with the measure $V_{l}^{(1 / 30)} l d l$. The inclusion of the scalar field leads to a slight decrease in the average volume and edge length, as well as a very slight decrease in $|\mathcal{R}|$. They again performed a fit for $\mathcal{R}$ according to (17) and found that the critical exponent and the location of $k_{c}$ were almost unchanged.

\subsection{Recovering the Newtonian potential}

Hamber and Williams [121] used an ansatz coming from a weak-field calculation for the amplitude of two gravitationally interacting particles separated by a fixed geodesic distance. They used the $l d l$-measure on a $16^{4}$-lattice, with $\lambda=1$ and $a=0$. The potential $V(d)$ is extracted from the connected Wilson-line to Wilson-line correlator. ¿From data in $d \in[0,5]$, and for sufficiently large $k$, one finds an attractive potential $V(d)<0$. They considered fits to various shapes

Living Reviews in Relativity (1998-13)

http://www.livingreviews.org 
of the potential $V$, in order to extract an effective Newton's constant. - They also suggested an effective action for $\mathcal{R}$ depending on $k, \lambda$ and $a$ that shares some of the features observed in the simulations.

\subsection{Gauge invariance in Regge calculus?}

A view that has been expressed frequently is that away from configurations with special symmetries, different edge length assignments correspond to inequivalent geometries, and in this sense Regge calculus possesses no gauge invariance ([187, 81, 124]; for a dissenting opinion, see [184]).

The diffeomorphism invariance can be recovered in a weak-field perturbation about flat space, as was shown by Roček and Williams [181, 183], and there is some evidence for the existence of analogous zero-modes in perturbations of regular, non-flat tesselations, at least in $2 \mathrm{~d}$ [122]. Inspired by the perturbative analysis around flat space, Hamber and Williams [122] argue that a similar gauge invariance should persist even if one perturbs around an arbitrary non-flat background. They propose as a possible definition for such gauge transformations local variations $\delta l_{i}^{2}$ of the link lengths that leave both the local volume and the local curvature terms invariant.

One may hope that in the non-perturbative Regge regime no gauge-fixing is necessary, since the contributions from zero-modes cancel out in the pathintegral representation for operator averages [124, 122]. Menotti and Peirano [162, 164, 163, 161], following a strategy suggested by Jevicki and Ninomiya [134], have argued vigorously that the functional integral should contain a nontrivial Faddeev-Popov determinant. Their starting point is somewhat different from that adopted in the path-integral simulations (see also [199]). They treat piecewise flat spaces as special cases of differentiable manifolds (with singular metric), with the action of the full diffeomorphism group still well-defined. To arrive at a concrete representation for the Faddeev-Popov term which could be used in simulations seems at present out of reach.

Recently, Hamber and Williams $[122,123]$ have argued that the $l d l$-lattice measure is the essentially unique local lattice measure over squared edge lengths (this is a special case of the one-parameter family of local measures of the form $\prod_{\sigma}[V(\sigma)]^{\nu} \prod_{l} l d l$; see also [24] for a related derivation). It does of course require a term with positive cosmological term in the action in order to suppress long edge lengths.

\subsection{Assorted topics}

Hartle $[124,125]$ has suggested computing the wave functional of the universe in a simplicial approximation, and evaluating the discrete path integral semiclassically near stationary points of the Regge action. He investigated numerically the extrema of the action (12) on small simplicial manifolds with topologies $S^{4}$, $\mathbb{C} P^{2}$ and $S^{2} \times S^{2}$ [126]. The properties of a Hartle-Hawking wave functional for a small complex with an $S^{3}$-boundary were studied in [127].

Living Reviews in Relativity (1998-13)

http://www.livingreviews.org 
Fröhlich [104] has advocated the need for a proof of reflection positivity of the Regge path integral, which one may expect to play a role in proving the unitarity of the theory. This can be formulated as a condition on the pathintegral measure (including the action) under the gluing of two simplicial fourmanifolds along a three-dimensional boundary.

Other authors have suggested associating gauge-theoretic instead of metric variables with the building blocks of a simplicial complex, for the case of the Poincaré group [71], the Lorentz group [140], and for Ashtekar gravity with gauge group $S U(2, \mathbb{C})[131,132]$, and reformulating the quantum theory in terms of them.

Hamiltonian 3+1 versions of Regge calculus have been studied classically (see [190] for a review), but attempts to quantize them have not progressed very far. One meets problems with the definition of the constraints and the (non-)closure of their Poisson algebra. A recent proposal for constructing a canonical quantum theory is due to Mäkelä [158], who constructed a simplicial version of the Wheeler-DeWitt equation, based on the use of area instead of length variables (which however are known to be overcomplete). In a similar vein, Khatsymovsky [141] has suggested that the operators measuring spatial areas ought to have a discrete spectrum.

\subsection{Summary}

Quantum Regge calculus is based on the well-explored classical discretization of the Einstein action due to Regge. Its weak-field limit around flat space agrees with the continuum result. Numerical simulations of the Euclidean path integral indicate the existence of a well-defined phase with small (negative) average curvature for sufficiently small $k$ and sufficiently large $\lambda$, even in the absence of higher-order curvature terms. Hamber [111, 113] has found some evidence for a second-order phase transition in the presence of a small higherorder derivative coupling, with a vanishing average curvature at the transition point. These findings have not been confirmed by other groups. The recent controversy in the dynamical triangulations approach teaches us to treat this issue with some caution.

Almost all simulations have been done on hypercubic, subdivided lattices with $T^{4}$-topology, which may introduce a systematic bias in the results. There is evidence that the choice of measure plays a role in the appearance and suppression of singular geometries, so-called spikes. The study of irregular lattices suggests a direct link of the transition points with the appearance of such spikes. This feature is reminiscent of the appearance of singular structures in dynamical triangulations. The coupling of a single scalar or $\mathrm{SU}(2)$-gauge field seems to have little influence on the phase structure of the gravitational sector.

Living Reviews in Relativity (1998-13)

http://www.livingreviews.org 


\section{Dynamical triangulations}

\subsection{Introduction}

This quantization approach has received a lot of attention since the early nineties $[2,1,11]$, inspired by analogous studies in two-dimensional gravity, where dynamical triangulation methods have been a valuable tool in complementing analytical results (see, for example, [85, 5]). I will here exclusively concentrate on the $4 \mathrm{~d}$ results. Other overview material is contained in $[6,136,195,4,3,17,59$, 73, 144, 135, 8, 9, 54].

Dynamical triangulations are a variant of quantum Regge calculus, where the dynamical variables are not the edge lengths of a given simplicial complex, but its connectivity. A precursor is Weingarten's [193] prescription for computing transition amplitudes between three-geometries, by summing over all interpolating four-geometries, built from equilateral 4d hypercubes living on an imbedding $p$-dimensional hypercubic lattice with lattice spacing $a$. Evaluating the Einstein action on such a configuration amounts to a simple counting of hypercubes of dimension 2 and 4, c.f. (21).

To avoid a potential overcounting in the usual Regge calculus, Römer and Zähringer [184] proposed a gauge-fixing procedure for Regge geometries. They argued for an essentially unique association of Riemannian manifolds and equilateral triangulations that in a certain sense are best approximations to the continuum manifolds. The resulting "rigid Regge calculus" is essentially the same structure that nowadays goes by the name of "dynamical triangulations". In this ansatz one studies the statistical mechanical ensemble of triangulated four-manifolds with fixed edge lengths, weighted by the Euclideanized Regge action, with a cosmological constant term, and optionally higher-derivative contributions. Each configuration represents a discrete geometry, i.e. the discrete version of a Riemannian four-metric modulo diffeomorphisms. At least for fixed total volume, the state sum converges for appropriate values of the bare coupling constants, if one restricts the topology (usually to that of a sphere $S^{4}$ ).

\subsection{Path integral for dynamical triangulations}

Denoting by $\mathcal{T}$ the set of all triangulations of the four-sphere, the partition function for the model is given by

$$
Z\left(\kappa_{2}, \kappa_{4}\right)=\sum_{T \in \mathcal{T}} \frac{1}{C(T)} e^{-S[T]}, \quad S[T]=-\kappa_{2} N_{2}(T)+\kappa_{4} N_{4}(T),
$$

where $N_{2}$ and $N_{4}$ denote the numbers of 2- and 4-simplices contained in the simplicial manifold $T$, and $C(T)$ is the order of the automorphism group of $T$. One may think of (21) as a grand canonical ensemble, with chemical potential $\kappa_{4}$. It is related to the canonical ensemble with fixed volume, $Z\left(\kappa_{2}, N_{4}\right)$, by a Legendre transform

$$
Z\left(\kappa_{2}, \kappa_{4}\right)=\sum_{N_{4}} e^{-\kappa_{4} N_{4}} Z\left(\kappa_{2}, N_{4}\right) \equiv \sum_{N_{4}} e^{-\kappa_{4} N_{4}} \sum_{T \in \mathcal{T}\left(N_{4}\right)} e^{\kappa_{2} N_{2}(T)}
$$


The metric information is encoded in the connectivity of the simplicial decomposition, since the individual 4 -simplices are assumed equilateral, with the edge length $a$ set to 1 .

To understand the simple form of the action $S$, recall that the curvature term in Regge calculus (c.f.(12) is represented by $\sum_{b} 2 \delta_{b} A_{b}$, which for fixed edge length is proportional to $\left(c_{4} N_{2}-10 N_{4}\right)$. The constant $c_{4}=2 \pi / \arccos \frac{1}{4}=$ 4.767 is determined from the condition that a triangulation of flat space should have average vanishing curvature $[2,11]$. (Because the four-simplices $\sigma$ are equilateral, zero curvature can only be achieved upon averaging. This explains the absence of a conventional perturbation theory around flat space.) The cosmological term is represented by $\lambda N_{4} V(\sigma) \sim \lambda N_{4}$. It is sometimes convenient to re-express $N_{2}$ as a function of $N_{0}$, using $N_{2}=2 N_{0}+2 N_{4}-4$, valid for the $S^{4}$ topology. The corresponding partition function is $Z\left(\kappa_{0}, \kappa_{4}\right)$ (where $\left.\kappa_{0}=2 \kappa_{2}\right)$.

\subsection{Existence of an exponential bound?}

As a consequence of identities and inequalities satisfied by the $N_{i}$ ([8] contains a detailed discussion), the action (21) is bounded above and below for fixed volume $N_{4}$. If the number of configurations for fixed $N_{4}$, is exponentially bounded as $N_{4} \rightarrow \infty$, that is, $Z\left(\kappa_{2}, N_{4}\right)$ grows at most as $Z\left(\kappa_{2}, N_{4}\right) \sim e^{\text {const } N_{4}}$, there is a "critical line" $\kappa_{4}=\kappa_{4}^{c}\left(\kappa_{2}\right)$ in the $\left(\kappa_{2}, \kappa_{4}\right)$-plane, where for fixed $\kappa_{2}, Z\left(\kappa_{2}, \kappa_{4}\right)$ converges for $\kappa_{4}>\kappa_{4}^{c}\left(\kappa_{2}\right)$. True critical behaviour may be found by approaching suitable points on this line from the region above the line, where $Z$ is well defined.

Doubts on the existence of an exponential bound were raised by Catterall et al [74], who considered the behaviour of $\Omega$ in $Z\left(\kappa_{0}, \kappa_{4}\right)=\sum_{N_{4}} e^{-\kappa_{4} N_{4}} \Omega\left(\kappa_{0}, N_{4}\right)$. Their data (taken for $N_{4} \leq 32 k$ ) were consistent with a leading factorial behaviour $\Omega \sim\left(N_{4} !\right)^{\delta}$. The same scenario was favoured by de Bakker and Smit [89], who performed further investigations of $\kappa_{4}^{c}$. Subsequently, Ambjørn and Jurkiewicz [12] and Brügmann and Marinari [60] added further data points at $N_{4}=64 k$ and $N_{4}=128 k$ respectively. Their numerical results, as well as those by Catterall et al [78], who employed an alternative method for measuring $\Omega$, favour the existence of an exponential bound, although they cannot claim to be conclusive.

There have also been theoretical arguments for the existence of an exponential bound, based on the proofs of such bounds for the counting of minimal geodesic ball coverings of Riemannian spaces of bounded geometry [68, 26], and the counting of discrete curvature assignments to unordered sets of bones [8].

\subsection{Performing the state sum}

The partition function is evaluated numerically with the help of a Monte-Carlo algorithm (see [57, 50, 72] for details). There is a set of five topology-preserving moves which change a triangulation locally, and which are ergodic in the grand canonical ensemble [171, 172, 105] (see also [8] for a discussion). No ergodic finite set of topology- and volume-preserving moves exists for generic four-dimensional manifolds. This prevents one from using the canonical (i.e. volume-preserving)

Living Reviews in Relativity (1998-13)

http://www . livingreviews .org 
ensemble. If $S^{4}$ is not algorithmically recognizable in the class of all piecewise linear (or smooth) 4d manifolds, the numerical simulations may miss out a substantial part of the state space because of the absence of "computational ergodicity" [170].

An attempt was made by Ambjørn and Jurkiewicz [13] to link the nonrecognizability to the presence of large- $N_{4}$ barriers, which should manifest themselves as an obstacle to cooling down an large initial random triangulation to the minimal $S^{4}$-configuration. No such barriers were found for system sizes $\leq 64 k$, but unfortunately they were also absent for an analogous simulation (for $N_{5} \leq 32 k$ ) performed by de Bakker [86] for $S^{5}$, which is not recognizable.

Since the local moves alter the volume, one works in practice with a "quasicanonical" ensemble, i.e. one uses the grand canonical ensemble $Z\left(\kappa_{2}, \kappa_{4}\right)$, but adds a potential term to the action so that the only relevant contributions come from states in an interval $\left[V-\Delta N_{4}, V+\Delta N_{4}\right]$ around the target volume $V$. There have been several cross-checks which have found no dependence of the results on the width and shape of the potential term [79, 51], but the lattice sizes and fluctuations may still be too small to detect a potential failure of ergodicity, c.f. [53].

To improve the efficiency of the algorithm, Ambjørn and Jurkiewicz [14] used additional global (topology-preserving) "baby universe surgery" moves, by cutting and gluing pieces of the simplicial complex. In the branched polymer phase, one can estimate the entropy exponent $\gamma$, assuming a behaviour of the form $Z\left(\kappa_{2}, N_{4}\right)=N_{4}^{\gamma\left(\kappa_{2}\right)-3} e^{\kappa_{4}^{c}\left(\kappa_{2}\right) N_{4}} \times\left(1+O\left(1 / N_{4}\right)\right)$, by counting baby universes of various sizes. At the transition point, one finds $\gamma\left(\kappa_{2}^{c}\right) \approx 0$ [10, 14, 97]. More recently, Egawa et al [98] have reported a value of $\gamma \approx 0.26$.

\subsection{The phase structure}

Already the first simulations by Agishtein and Migdal [2, 1] and Ambjørn and Jurkiewicz [11] of dynamically triangulated $4 \mathrm{~d}$ gravity exhibited a clear twophase structure. After tuning to the infinite-volume or critical line $\kappa_{4}^{c}\left(\kappa_{2}\right)$, one identifies two regions, $\kappa_{2}<\kappa_{2}^{c}$ and $\kappa_{2}>\kappa_{2}^{c}$. The critical value $\kappa_{2}^{c}$ depends on the volume $N_{4}$, and it was conjectured that it may move out to $+\infty$ as $N_{4} \rightarrow \infty[75,89]$, but it was later shown to converge to a finite value [14]. One can characterize the region with small $\kappa_{2}<\kappa_{2}^{c}$ as the hot, crumpled, or condensed phase. It has small negative or positive curvature, large (possibly infinite) Hausdorff dimension $d_{H}$ and a high connectivity. By contrast, for $\kappa_{2}>\kappa_{2}^{c}$ one is in the cold, extended, elongated, or fluid phase. It has large positive curvature, with an effective tree-like branched-polymer geometry, and $d_{H} \approx 2$.

The location of the critical point on the infinite-volume line may be estimated from the peak in the curvature susceptibility $\chi\left(\kappa_{2}, N_{4}\right)=\left(\left\langle N_{2}^{2}\right\rangle-\left\langle N_{2}\right\rangle^{2}\right) / N_{4}$ $[2,1,11,192,129,90]$, or the node susceptibility $\chi\left(\kappa_{0}, N_{4}\right)=\left(\left\langle N_{0}^{2}\right\rangle-\left\langle N_{0}\right\rangle^{2}\right) / N_{4}$ $[75,76]$, as well as higher cumulants of $N_{0}$ [48]. In [14], it was suggested that one may alternatively estimate $\kappa_{2}^{c}\left(N_{4}\right)$ by looking at the behaviour of the entropy exponent $\gamma$, approaching $\kappa_{2}^{c}$ from the elongated phase. More recently, Catterall 
et al [77] have used the fluctuations $\chi_{0}=\left(\left\langle\omega_{0}^{2}\right\rangle-\left\langle\omega_{0}\right\rangle^{2}\right) / N_{4}$ of the local volume $\omega_{0}$ around singular vertices as an order parameter.

\subsection{Evidence for a second-order transition?}

It sometimes seems to be assumed that if one were to find a continuum theory at a second-order phase transition, it would have flat Minkowski space as its ground state (in spite of the $S^{4}$-topology), and gravitonic spin-2 excitations. An alternative scenario with a constant-curvature sphere-metric has been put forward by de Bakker and Smit [90, 92].

Agishtein and Migdal [2] initially reported a hysteresis in the average curvature $\langle R\rangle\left(\kappa_{2}\right)$, indicating a first-order transition. However, subsequent authors $[11,191,192,75,76]$ found numerical data not incompatible with the existence of a second-order transition, and also Agishtein and Migdal [1] retracted their original claim as a result of a closer examination of the fixed point region.

One tries to discriminate between a first- and second- (or higher-)order transition by looking at the Binder parameter [191, 192, 7], or scaling exponents $\alpha$ governing the scaling behaviour $\sim\left|\kappa_{2}^{c}-\kappa_{2}\right|^{\alpha}$ of suitable observables [1, 192], or the peak height of susceptibilities as a function of the volume $N_{4}$ $[16,75,76,14,48,87]$. Other scaling relations are discussed in [90, 91, 96, 95]. However, since critical parameters are hard to measure, and it is difficult to estimate finite-size effects, none of the data can claim to be conclusive.

Some doubts were cast on the conjectured continuous nature of the phase transition by Bialas et al [48], who found an unexpected two-peak structure in the distribution of nodes near the fixed point. This was strengthened further by data taken at $64 \mathrm{k}$ by de Bakker [87], with an even more pronounced double peak (see also [51]). Most likely previous simulations were simply too small to detect the true nature of the phase transition. Both Bialas et al and de Bakker observed that the finite size scaling exponents extracted from the node susceptibility $\chi_{0}$ grow with volume and may well reach the value 1 expected for a first-order transition as $V \rightarrow \infty$.

The origin of this behaviour was further elucidated by Bialas et al [49, 47] and Bialas and Burda [46], who found a simple mean-field model that reproduces qualitatively the phase structure of $4 \mathrm{~d}$ dynamically triangulated quantum gravity. With an appropriate choice of local weights, this model has a condensed and a fluid phase, with a first-order transition in between. A similar behaviour was found by Catterall et al [77], who made a related mean-field ansatz, with the local weights depending on the local entropies around the vertices.

\subsection{Influence of the measure}

The inclusion of a local measure term corresponding to $(\operatorname{det} g)^{\frac{\mu}{2}}$ was studied by Brügmann and Marinari $[56,57,58]$. This amounts to adding to the action a term of the form $-\mu \sum_{v} \ln o(v) / 5$, where $o(v)$ is the number of 4 -simplices containing a vertex $v$. For $\mu=-5,-1,1,5$, this seems to lead merely to a shift

Living Reviews in Relativity (1998-13)

http://www . livingreviews .org 
of the critical line. This setting has been revived recently by Renken $[178,177]$ in the context of a renormalization group analysis.

\subsection{Higher-derivative terms}

Higher-order derivative terms were considered by Ambjørn et al [16] (see also [143]), who added a term of the form

$$
h c_{4}^{-2} \sum_{b} o(b)\left(\frac{c_{4}-o(b)}{o(b)}\right)^{2}
$$

to the action, where again $c_{4}=4.767$ and $o(b)$ denotes the number of 4 -simplices sharing a bone $b$. For $h \in[0,20]$, and with volumes up to $32 k$, no major qualitative changes of the geometrical observables were found. The inclusion of the higher-derivative term also does not improve the behaviour of the average curvature $\langle R\rangle$, which continues to be positive at the critical point, whereas from a naïve comparison with the continuum theory one would expect it to scale to zero. (This is also incompatible with the prediction of Antoniadis et al [20], should dynamical triangulations possess an infrared stable fixed point.) De Bakker and Smit [89] have argued that this may not be a reason of concern, since one expects the volume and curvature terms to mix under renormalization.

\subsection{Coupling to matter fields}

Ambjørn et al [7] considered the influence of both Ising spins and Gaussian scalar fields on bulk geometric quantities. The phase structure remained essentially unchanged, and no improvement in the scaling behaviour of $\langle R\rangle$ was found. Taken together with their results on higher-derivative gravity [16], one finds a universal linear dependence of the cosmological constant $\kappa_{4}^{c}\left(\kappa_{2}\right)$, with slope $\sim 2.5$.

Coupling to $\mathbb{Z}_{2}$-spin variables $s(l)$ located on edges $l$ was considered by Ambjørn et al [15], who added a Wilson loop term $S_{\mathrm{W}}[s ; T]=-\beta \sum_{b \in T} o(b)$ $\prod_{l \in b} s(l)$ to the action. The matter sector behaves largely as expected when $\kappa_{2}$ is varied between the crumpled and the elongated gravity phase. However, in the common critical region of both sectors, where a priori one might have expected interesting effects, the critical behaviour seems to agree with that of the pure gravity system.

More recently, Bilke et al [52] have reported a non-trivial back-reaction of matter on geometry, when considering coupling to several non-compact $U(1)$ gauge fields. Their study was in part motivated by a continuum analysis of the dynamics of the conformal factor of Antoniadis et al [21]. Including three fields $U(1)$-fields seems to lead to a total suppression of the branched polymer phase, which is replaced by a new weak-coupling phase with negative susceptibility exponent $\gamma$ and a fractal dimension $\approx 4$. These are clearly interesting results, but should be treated with some caution because of the small lattice sizes involved $\left(N_{4} \leq 16 k\right)$. 


\subsection{Non-spherical lattices}

Alternative topologies for the underlying simplicial complex were considered by Bilke et al $[51,53]$. They showed that for topologies $S^{1} \times S^{3}$ and $T^{4}$, the free energy agrees to leading order with that of $S^{4}$. Neither the critical value $\kappa_{4}^{c}\left(\kappa_{2}, N_{4}\right)$, nor the leading contributions to the action density $\left\langle N_{2}\right\rangle / N_{4}$ are changed. However, the simulations become more involved, since the size of the minimal configurations is increased.

\subsection{Singular configurations}

The singular nature of the geometry in the phase below the critical value $\kappa_{2}^{c}$ can be quantified by the distribution $\rho(n)$ of the vertex order $o(v)$,

$$
\rho(n)=\frac{1}{N_{0}}\left\langle\sum_{v} \delta_{o(v), n}\right\rangle,
$$

first considered by Hotta et al [129]. It has a continuum part and a separate peak at high vertex order. One can suppress this effect by adding a term $\sum_{v}(o(v)-5)^{2}$ to the action (see also [19] for a discussion of terms of a similar nature), but this leads to a simultaneous disappearance of the phase transition. Hotta et al [130] have checked that for a variety of initial configurations the singular structure is a generic feature of the model.

Catterall et al $[79,80]$ observed that the pair of singular vertices form the end points of a singular link. They also offered a possible explanation for the formation of these singular structures: Simplices of sufficiently low dimension can maximize their local entropy by acquiring large local volumes (see also [49] for a mean-field argument). Catterall et al [77] found two pseudo-critical points, $\kappa_{0}^{(1)}$ and $\kappa_{0}^{(2)}$, associated with the creation of singular vertices and links, which seem to merge into a single critical point $\kappa_{0}^{c}$ as $V \rightarrow \infty$. One concludes that the observed phase transition in the $4 \mathrm{~d}$ dynamical triangulations model is driven by the appearance and disappearance of singular geometries.

\subsection{Renormalization group}

There have been attempts to apply renormalization group techniques, assuming that the transition is indeed continuous. Burda et al [61] and later Bialas et al [48] considered the "cutting of the last generation of minimal-neck baby

universes". This step can only be performed once, which severely limits the power of the procedure. Renken [178, 177] has applied a different blocking move, involving node deletion, and studied the RG flow using the volume and the vertex order as observables.

\subsection{Exploring geometric properties}

Since the effective geometry in both phases is rather singular, different ways of measuring length may lead to inequivalent definitions of "dimension". A

Living Reviews in Relativity (1998-13)

http://www.livingreviews.org 
common (local) notion is derived from the volume of a geodesic ball with radius $r$. Usually the radius is measured in terms of the geodesic distance $d_{1}$ (the minimal number of links), or dual geodesic distance $d_{4}$ (the minimal number of links of the dual graph). Alternatively, one may consider the number $n(r)$ of 4-simplices in spherical shells of thickness 1 at distance $r$, and define a fractal dimension by $\langle n(r)\rangle \sim r^{d_{\mathrm{F}}-1}[90,14]$.

To extract a global length scale, one may use the averages $\left\langle d_{1}\right\rangle,\left\langle d_{4}\right\rangle$, or consider the average "radius of the universe" $\langle r(T)\rangle$ [11] to obtain a cosmological Hausdorff dimension $d_{\mathrm{CH}}$, or the "average intrinsic linear extent" $L=$ $\left\langle V^{-2} \sum_{i, j} d_{4}(i, j, T)\right\rangle$ (see, for example, [75, 76]). Away from the phase transition, the fractal dimensions associated with these geometric construction are more or less equivalent and give $d_{\mathrm{F}}=\infty$ in the crumpled phase and $d_{\mathrm{F}} \approx 2$ in the elongated phase. It is difficult to measure the dimension close to the transition point.

It will not be straightforward to interpret the behaviour of observables (defined in analogy with the continuum theory), since in most of the phase space the geometry of the simplicial complex is far from approximating a metric 4manifold. In search of a semiclassical interpretation for geometric observables, an alternative notion of local curvature for a simplicial manifold was suggested by de Bakker and Smit [90], based on a continuum expansion of the volume of a geodesic ball. Assuming furthermore that independent of $n, n$-volumes of balls with radius $r$ behave like regions on $S^{n} \subset \mathbb{R}^{n+1}$, they extracted scaling relations for various geometric quantities for an intermediate range for $r$. This line of thought was pursued further in [185].

Close to the phase transition, one may investigate the behaviour of test particles (ignoring back-reactions on the geometry). Comparing the mass extracted from the one-particle propagator with the energy of the combined system obtained from the two-particle propagator [88, 92, 93], one does indeed find evidence for gravitational binding.

\subsection{Two-point functions}

It is possible to define two-point correlation functions on random geometries $[16,14]$, which are to be thought of as the discrete analogues of formal continuum correlators

$G_{\mathcal{O}}(r)=\int_{\substack{\text { Riem } S^{4} \\ \text { Diff } S^{4}}} D g_{\mu} e^{-S} \int d^{4} x \int d^{4} x^{\prime} \sqrt{\operatorname{det} g(x)} \sqrt{\operatorname{det} g\left(x^{\prime}\right)} \mathcal{O}(x) \mathcal{O}\left(x^{\prime}\right) \delta\left(d_{g}\left(x, x^{\prime}\right)-r\right)$,

for local observables $\mathcal{O}(x)$, with $d_{g}$ denoting the geodesic distance with respect to the metric $g_{\mu \nu}$. There is an ambiguity in defining the connected part of the correlator (25), as was pointed out by de Bakker and Smit [91, 92]. Contrary to expectations, after subtraction of the square of the curvature expectation value, the resulting quantity $\langle R R\rangle(r)-\langle R\rangle^{2}$ does not scale to zero with large distances. They therefore proposed an alternative definition of the connected 
two-point function, by subtracting the square of a "curvature-to-nothing" correlator $\langle R\rangle(r)$. This definition was compared in more detail by Bialas [45] with a more conventional notion, as, for example, the one used in [48]. For the case of curvature correlators, their behaviour differs significantly, especially at short distances.

\subsection{Summary}

In the dynamical triangulations approach, one studies the properties of a statistical ensemble of simplicial four-geometries à la Regge of fixed edge lengths. By summing over such discrete configurations according to (21), one has implicitly assumed that this leads to a uniform sampling of the space of smooth Riemannian manifolds. There is no obvious weak-field limit, but this is no obstacle in principle to the path-integral construction. Numerical simulations indicate the existence of a well-defined phase for sufficiently small $\kappa_{2}$ (inverse Newton's constant) and a sufficiently large cosmological constant. For small $\kappa_{2}$, one finds a "crumpled" phase, with small average curvature and a large Hausdorff dimension, and for large $\kappa_{2}$ an elongated, effectively two-dimensional polymer phase. At present, the consensus seems to be that the corresponding phase transition is of first order, with a finite average curvature at the transition point.

Almost all simulations have been done on simplicial manifolds with $S^{4}$ topology. Neither the inclusion of factors of $(\operatorname{det} g)^{\rho}$ in the measure nor the addition of higher-order curvature terms to the action seem to have a substantial influence on the phase structure. Also matter coupling to spinorial and scalar fields does not seem to lead to a change of universality class, although the inclusion of several gauge fields may have a more drastic effect. The study of singular structures (vertices of high coordination number) has led to a qualitative understanding of the phase structure of the model.

Living Reviews in Relativity (1998-13)

http://www.livingreviews.org 


\section{Conclusions and Outlook}

In this review, I have collected a variety of results on discrete four-dimensional models of quantum gravity, mainly coming from Euclidean path-integral approaches. Numerical simulations have yielded information on the phase structure of these models, the behaviour of two-point functions and a number of other properties of their partition functions. All of the path-integral models have some qualitative features in common. They need a (sufficiently large, positive) cosmological constant $\lambda$ to be well defined. For sufficiently small values of Newton's constant $G$, one finds a phase of collapsed geometry, with effective dimension $<4$. In the gauge-theoretic model, the metric is degenerate; in Regge calculus, one finds spiky configurations; and in dynamical triangulations, the ensemble behaves like that of a branched polymer.

In all cases, one observes a transition on the boundary of this phase, but so far no convincing evidence of long-range correlations has been found in its vicinity. Within the accuracy of the numerical simulations, this main conclusion is not altered by the inclusion of determinantal factors $(\operatorname{det} g)^{\rho}$ in the measure, the inclusion of higher-order derivative terms, or the addition of matter fields. Why does this happen? Each of the approaches can claim that its state space represents, at least roughly, an approximation to the space of smooth Riemannian metrics or geometries. This leaves only the path-integral measure as a possible culprit. The measures used up to now were the simplest ones compatible with considerations of locality and gauge-invariance. It seems premature to blame the absence of diffeomorphism invariance (whose status in the gauge-theoretic formulation and the Regge calculus program remains unclear), since the explicitly diffeomorphism-invariant dynamical triangulations approach suffers from similar problems. Further analytical insights are needed to understand which modifications of the measure would make these models more interacting.

There are a number of loop holes which could change the picture just presented. It is possible that adding enough matter of the correct type could have a non-trivial effect, or that Regge calculus with the inclusion of higher-order curvature terms does indeed possess a second-order phase transition. Since we have very little experience with universality properties of $4 \mathrm{~d}$ generally covariant theories, it is not a priori clear whether the choice of measure and the initial restrictions on the lattice geometry can affect the final results.

One may of course take the attitude that something is fundamentally wrong with trying to construct a theory of quantum gravity via a statistical field theory approach, and that a different starting point is needed, an obvious candidate being a non-perturbative theory of superstrings, or of more general extended objects. In any case, these different approaches need not be mutually exclusive, and one may therefore take the results of the discrete approaches presented here as an indication that other attempts of constructing quantum gravity nonperturbatively may run into similar difficulties.

A further unresolved problem is the "analytic continuation" of the pathintegral results to Lorentzian signature. The Hamiltonian ansatz circumvents this problem, and some progress has been made in the Hamiltonian gauge-

Living Reviews in Relativity (1998-13)

http://www. livingreviews.org 
theoretic discrete approach. Although the kinematical structure is in place and some information on the constraint algebra has been obtained, the physical state space has not yet been identified. Its results are therefore not sufficiently complete to admit comparison with the path-integral simulations. For the simplicial formulations, only little is known about their canonical counterparts. One would hope that future research will throw further light on these issues.

Acknowledgement. I am indebted to R. Williams, P. Menotti, H. Hamber, W. Beirl and J. Ambjørn for comments and criticism, to E. Schlenk for help with the references, and to R. Helling for assistance in reformatting them.

Living Reviews in Relativity (1998-13)

http://www. livingreviews.org 


\section{References}

[1] Agishtein, M.E., and Migdal, A.A., "Critical behavior of dynamically triangulated quantum gravity in four-dimensions", Nucl. Phys. B, 385, 395-412, (1992). For a related online version see: M.E. Agishtein, et al., "Critical behavior of dynamically triangulated quantum gravity in fourdimensions", (April, 1992), [Online Los Alamos Archive Preprint]: cited on 19 November 1998, http://xxx.lanl.gov/abs/hep-lat/9204004. $4.1,4.5,4.6$

[2] Agishtein, M.E., and Migdal, A.A., "Simulations of four-dimensional simplicial quantum gravity as dynamical triangulation", Mod. Phys. Lett. A, 7, 1039-1061, (1992). 4.1, 4.2, 4.5, 4.6

[3] Ambjørn, J., "Quantum gravity represented as dynamical triangulations", Class. Quantum Grav., 12, 2079-2134, (1995). 4.1

[4] Ambjørn, J., "Recent progress in the theory of random surfaces and simplicial quantum gravity", Nucl. Phys. Proc. Suppl., 42, 3-16, (1995). For a related online version see: J. Ambjørn, "Recent progress in the theory of random surfaces and simplicial quantum gravity", (December, 1994), [Online Los Alamos Archive Preprint]: cited on 19 November 1998, http://xxx.lanl.gov/abs/hep-lat/9412006. 4.1

[5] Ambjørn, J., "Quantization of geometry", in David, F., Ginsparg, P., and Zinn-Justin, J., eds., Fluctuating geometries in statistical mechanics and field theory (Les Houches Summer School, Session LXII, 1994), 77-193, (Elsevier, Amsterdam, 1996). For a related online version see: J. Ambjørn, "Quantization of geometry", (November, 1994), [Online Los Alamos Archive Preprint]: cited on 19 November 1998, http: //xxx.lanl.gov/abs/hep-th/9411179. 4.1

[6] Ambjørn, J., Burda, Z., Jurkiewicz, J., and Kristjansen, C.F., "Quantum gravity represented as dynamical triangulations", Acta Phys. Pol. B, 23, 991-1030, (1992). 4.1

[7] Ambjørn, J., Burda, Z., Jurkiewicz, J., and Kristjansen, C.F., "Fourdimensional dynamically triangulated gravity coupled to matter", Phys. Rev. D, 48, 3695-3703, (1993). For a related online version see: J. Ambjørn, et al., "Four-dimensional dynamically triangulated gravity coupled to matter", (March, 1993), [Online Los Alamos Archive Preprint]: cited on 19 November 1998, http://xxx.lanl.gov/abs/ hep-th/9303042. 4.6, 4.9

[8] Ambjørn, J., Carfora, M., and Marzuoli, A., The geometry of dynamical triangulations, volume m50 of Lecture Notes in Physics, New Series, (Springer, Berlin, 1997). For a related online version see: J. Ambjørn, et al., "The geometry of dynamical triangulations", (December, 1996), 
[Online Los Alamos Archive Preprint]: cited on 19 November 1998, http://xxx.lanl.gov/abs/hep-th/9612069. 3.1, 4.1, 4.3, 4.4

[9] Ambjørn, J., Durhuus, B., and Jonsson, T., Quantum geometry, (Cambridge University Press, Cambridge, 1997). 4.1

[10] Ambjørn, J., Jain, S., Jurkiewicz, J., and Kristjansen, C.F., "Observing 4-d baby universes in quantum gravity", Phys. Lett. B, 305, 208-213, (1993). For a related online version see: J. Ambjørn, et al., "Observing 4d baby universes in quantum gravity", (March, 1993), [Online Los Alamos Archive Preprint]: cited on 19 November 1998, http://xxx.lanl.gov/ abs/hep-th/9303041. 4.4

[11] Ambjørn, J., and Jurkiewicz, J., "Four-dimensional simplicial quantum gravity", Phys. Lett. B, 278, 42-50, (1992). 4.1, 4.2, 4.5, 4.6, 4.13

[12] Ambjørn, J., and Jurkiewicz, J., "On the exponential bound in fourdimensional simplicial gravity", Phys. Lett. B, 335, 355-358, (1994). For a related online version see: J. Ambjørn, et al., "On the exponential bound in four-dimensional simplicial gravity", (May, 1994), [Online Los Alamos Archive Preprint]: cited on 19 November 1998, http: //xxx.lanl.gov/abs/hep-lat/9405010. 4.3

[13] Ambjørn, J., and Jurkiewicz, J., "Computational ergodicity of $S^{4}$ ", Phys. Lett. B, 345, 435-440, (1995). For a related online version see: J. Ambjørn, et al., "Computational ergodicity of $S^{4}$ ", (November, 1994), [Online Los Alamos Archive Preprint]: cited on 19 November 1998, http://xxx. lanl.gov/abs/hep-lat/9411008. 4.4

[14] Ambjørn, J., and Jurkiewicz, J., "Scaling in four dimensional quantum gravity", Nucl. Phys. B, 451, 643-676, (1995). For a related online version see: J. Ambjørn, et al., "Scaling in four dimensional quantum gravity", (March, 1995), [Online Los Alamos Archive Preprint]: cited on 19 November 1998, http://xxx.lanl.gov/abs/hep-th/9503006. 4.4, 4.5, 4.6, $4.13,4.14$

[15] Ambjørn, J., Jurkiewicz, J., Bilke, S., Burda, Z., and Petersson, B., "Z(2) gauge matter coupled to 4-d simplicial quantum gravity", Mod. Phys. Lett. A, 9, 2527-2542, (1994). 4.9

[16] Ambjørn, J., Jurkiewicz, J., and Kristjansen, C.F., "Quantum gravity, dynamical triangulation and higher derivative regularization", Nucl. Phys. $B, 393,601-632$, (1993). For a related online version see: J. Ambjørn, et al., "Quantum gravity, dynamical triangulation and higher derivative regularization", (August, 1992), [Online Los Alamos Archive Preprint]: cited on 19 November 1998, http://xxx.lanl.gov/abs/hep-th/9208032. $4.6,4.8,4.9,4.14$

Living Reviews in Relativity (1998-13)

http://www.livingreviews.org 
[17] Ambjørn, J., Jurkiewicz, J., and Watabiki, Y., "Dynamical triangulations, a gateway to quantum gravity?", J. Math. Phys., 36, 6299-6339, (1995). For a related online version see: J. Ambjørn, et al., "Dynamical triangulations, a gateway to quantum gravity?", (March, 1995), [Online Los Alamos Archive Preprint]: cited on 19 November 1998, http://xxx.lanl.gov/abs/hep-th/9503108. 4.1

[18] Ambjørn, J., Nielsen, J.L., Rolf, J., and Savvidy, G., "Spikes in quantum Regge calculus", Class. Quantum Grav., 14, 3225-3241, (1997). For a related online version see: J. Ambjørn, et al., "Spikes in quantum Regge calculus", (April, 1997), [Online Los Alamos Archive Preprint]: cited on 19 November 1998, http://xxx.lanl.gov/abs/gr-qc/9704079. 3.1

[19] Ambjørn, J., Savvidy, G.K., and Savvidy, K.G., "Alternative actions for quantum gravity and the intrinsic rigidity of the space-time", Nucl. Phys. $B$, 486, 390-412, (1997). For a related online version see: J. Ambjørn, et al., "Alternative actions for quantum gravity and the intrinsic rigidity of the space-time", (June, 1996), [Online Los Alamos Archive Preprint]: cited on 19 November 1998, http://xxx.lanl.gov/abs/ hep-th/9606140. 3.2, 4.11

[20] Antoniadis, I., Mazur, P.O., and Mottola, E., "Scaling behavior of quantum four-geometries", Phys. Lett. B, 323, 284-291, (1994). For a related online version see: I. Antoniadis, et al., "Scaling behavior of quantum fourgeometries", (January, 1993), [Online Los Alamos Archive Preprint]: cited on 19 November 1998, http://xxx.lanl.gov/abs/hep-th/9301002. 4.8

[21] Antoniadis, I., Mazur, P.O., and Mottola, E., "Criticality and scaling in 4d quantum gravity", Phys. Lett. B, 394, 49-56, (1997). For a related online version see: I. Antoniadis, et al., "Criticality and scaling in $4 \mathrm{~d}$ quantum gravity", (November, 1996), [Online Los Alamos Archive Preprint]: cited on 19 November 1998, http://xxx.lanl.gov/abs/hep-th/9611145. 4.9

[22] Ashtekar, A., "New variables for classical and quantum gravity", Phys. Rev. Lett., 57, 2244-2247, (1986). 2.9

[23] Ashtekar, A., "A new Hamiltonian formulation of general relativity", Phys. Rev. D, 36, 1587-1603, (1987). 2.9

[24] Bander, M., "Functional measure for lattice gravity", Phys. Rev. Lett., $\mathbf{5 7}, \mathbf{1 8 2 5}-1827,(1986) . \quad 3.13$

[25] Barbero G., J.F., "Real Ashtekar variables for Lorentzian signature space-times", Phys. Rev. D, 51, 5507-5510, (1995). For a related online version see: J.F. Barbero G., "Real Ashtekar variables for Lorentzian signature space-times", (October, 1994), [Online Los Alamos Archive 
Preprint]: cited on 19 November 1998, http://xxx.lanl.gov/abs/ gr-qc/9410014. 2.16

[26] Bartocci, C., Bruzzo, U., Carfora, M., and Marzuoli, A., "Entropy of random coverings and 4D quantum gravity", J. Geom. Phys., 18, 247294, (1996). For a related online version see: C. Bartocci, et al., "Entropy of random coverings and 4D quantum gravity", (December, 1994), [Online Los Alamos Archive Preprint]: cited on 19 November 1998, http://xxx. lanl.gov/abs/hep-th/9412097. 4.3

[27] Beirl, W., Berg, B.A., Krishnan, B., Markum, H., and Riedler, J., "Static quark potentials in quantum gravity", Phys. Lett. B, 348, 355-359, (1995). For a related online version see: W. Beirl, et al., "Static quark potentials in quantum gravity", (February, 1995), [Online Los Alamos Archive Preprint]: cited on 19 November 1998, http://xxx.lanl.gov/abs/ hep-lat/9502006. 3.10, 3.10

[28] Beirl, W., Berg, B.A., Krishnan, B., Markum, H., and Riedler, J., "SU(2) potentials in quantum gravity", Nucl. Phys. Proc. Suppl., 42, 707-709, (1995). For a related online version see: W. Beirl, et al., "SU(2) potentials in quantum gravity", (December, 1994), [Online Los Alamos Archive Preprint]: cited on 19 November 1998, http://xxx.lanl.gov/abs/ hep-lat/9412037. 3.10, 3.10

[29] Beirl, W., Gerstenmayer, E., and Markum, H., "Exploration of simplicial quantum gravity in four dimensions", Nucl. Phys. Proc. Suppl., 26, 575577, (1992). 3.5

[30] Beirl, W., Gerstenmayer, E., and Markum, H., "Influence of the measure on simplicial quantum gravity in four-dimensions", Phys. Rev. Lett., 69, 713-716, (1992). For a related online version see: W. Beirl, et al., "Influence of the measure on simplicial quantum gravity in four-dimensions", (April, 1992), [Online Los Alamos Archive Preprint]: cited on 19 November 1998, http://xxx.lanl.gov/abs/hep-lat/9204010. 3.5

[31] Beirl, W., Gerstenmayer, E., Markum, H., and Riedler, J., "Gravitational action versus entropy on simplicial lattices in four-dimensions", Nucl. Phys. Proc. Suppl., 30, 764-767, (1993). For a related online version see: W. Beirl, et al., "Gravitational action versus entropy on simplicial lattices in four-dimensions", (November, 1992), [Online Los Alamos Archive Preprint]: cited on 19 November 1998, http://xxx.lanl.gov/ abs/hep-lat/9211044. 3.7, 3.9

[32] Beirl, W., Gerstenmayer, E., Markum, H., and Riedler, J., "The well defined phase of simplicial quantum gravity in four-dimensions", Phys. Rev. $D, 49,5231-5239$, (1994). For a related online version see: W. Beirl, et al., "The well defined phase of simplicial quantum gravity in four-dimensions", (February, 1994), [Online Los Alamos Archive Preprint]: cited on 19 November 1998, http://xxx.lanl.gov/abs/hep-lat/9402002. 3.7

Living Reviews in Relativity (1998-13)

http://www.livingreviews.org 
[33] Beirl, W., Hauke, A., Homolka, P., Krishnan, B., Kröger, H., Markum, H., and Riedler, J., "The phase structure of pure Regge gravity", Nucl. Phys. Proc. Suppl., 47, 625-628, (1996). For a related online version see: W. Beirl, et al., "The phase structure of pure Regge gravity", (October, 1995), [Online Los Alamos Archive Preprint]: cited on 19 November 1998, http://xxx.lanl.gov/abs/hep-lat/9510021. 3.9

[34] Beirl, W., Hauke, A., Homolka, P., Markum, H., and Riedler, J., "Correlation functions in lattice formulations of quantum gravity", Nucl. Phys. Proc. Suppl., 53, 735-738, (1997). For a related online version see: W. Beirl, et al., "Correlation functions in lattice formulations of quantum gravity", (August, 1996), [Online Los Alamos Archive Preprint]: cited on 19 November 1998, http://xxx.lanl.gov/abs/hep-lat/9608055. 3.8, 3.9

[35] Beirl, W., Markum, H., and Riedler, J., "On the measure of simplicial quantum gravity in four-dimensions", in Carr, J., and Perrottet, M., eds., High energy physics: 1993 International Europhysics Conference, 214-216, (Éditions Frontières, Gif-sur-Yvette, 1994). For a related online version see: W. Beirl, et al., "On the measure of simplicial quantum gravity in four-dimensions", (September, 1993), [Online Los Alamos Archive Preprint]: cited on 19 November 1998, http: //xxx.lanl.gov/abs/hep-lat/9309008. 3.7

[36] Beirl, W., Markum, H., and Riedler, J., "Regge gravity on general triangulations", Phys. Lett. B, 341, 12-18, (1994). For a related online version see: W. Beirl, et al., "Regge gravity on general triangulations", (July, 1994), [Online Los Alamos Archive Preprint]: cited on 19 November 1998, http://xxx.lanl.gov/abs/hep-lat/9407020. 3.9

[37] Beirl, W., Markum, H., and Riedler, J., "Two point functions of fourdimensional simplicial quantum gravity", Nucl. Phys. Proc. Suppl., 34, 736-738, (1994). For a related online version see: W. Beirl, et al., "Two point functions of four-dimensional simplicial quantum gravity", (December, 1993), [Online Los Alamos Archive Preprint]: cited on 19 November 1998, http://xxx.lanl.gov/abs/hep-lat/9312053. 3.8

[38] Berg, B.A., "Exploratory numerical study of discrete quantum gravity", Phys. Rev. Lett., 55, 904-907, (1985). 3.3

[39] Berg, B.A., "Entropy versus energy on a fluctuating four-dimensional Regge skeleton", Phys. Lett. B, 176, 39-44, (1986). 3.3

[40] Berg, B.A., "Quantum gravity motivated computer simulations", in Mitter, H., and Widder, F., eds., Particle physics and astrophysics, 223-259, (Springer, Berlin, 1989). 3.1, 3.3

[41] Berg, B.A., Beirl, W., Krishnan, B., Markum, H., and Riedler, J., "Phase diagram of Regge quantum gravity coupled to SU(2) gauge theory", Phys. 
Rev. D, 54, 7421-7425, (1996). For a related online version see: B.A. Berg, et al., "Phase diagram of Regge quantum gravity coupled to $\mathrm{SU}(2)$ gauge theory", (May, 1996), [Online Los Alamos Archive Preprint]: cited on 19 November 1998, http://xxx.lanl.gov/abs/hep-lat/9605036. 3.10, 3.10

[42] Berg, B.A., and Krishnan, B., "Asymptotic freedom and Regge-Einstein quantum gravity", Nucl. Phys. Proc. Suppl., 30, 768-770, (1993). 3.10, 3.10

[43] Berg, B.A., and Krishnan, B., "Phase structure of SU(2) lattice gauge theory with quantum gravity", Phys. Lett. B, 318, 59-62, (1993). For a related online version see: B.A. Berg, et al., "Phase structure of SU(2) lattice gauge theory with quantum gravity", (June, 1993), [Online Los Alamos Archive Preprint]: cited on 19 November 1998, http://xxx. lanl.gov/abs/hep-lat/9306006. 3.10, 3.10

[44] Berg, B.A., Krishnan, B., and Katoot, M., "Asymptotic freedom and Euclidean quantum gravity", Nucl. Phys. B, 404, 359-384, (1993). For a related online version see: B.A. Berg, et al., "Asymptotic freedom and Euclidean quantum gravity", (September, 1992), [Online Los Alamos Archive Preprint]: cited on 19 November 1998, http://xxx.lanl.gov/ abs/hep-lat/9209001. 3.10, 3.10

[45] Bialas, P., "Correlations in fluctuating geometries", Nucl. Phys. Proc. Suppl., 53, 739-742, (1997). For a related online version see: P. Bialas, "Correlations in fluctuating geometries", (August, 1996), [Online Los Alamos Archive Preprint]: cited on 19 November 1998, http://xxx. lanl.gov/abs/hep-lat/9608029. 4.14

[46] Bialas, P., and Burda, Z., "Collapse of 4-d random geometries", Phys. Lett. B, 416, 281-285, (1998). For a related online version see: P. Bialas, et al., "Collapse of 4-d random geometries", (July, 1997), [Online Los Alamos Archive Preprint]: cited on 19 November 1998, http://xxx. lanl.gov/abs/hep-lat/9707028. 4.6

[47] Bialas, P., Burda, Z., and Johnston, D.A., "Balls in boxes and quantum gravity", Nucl. Phys. Proc. Suppl., 63, 763-765, (1998). For a related online version see: P. Bialas, et al., "Balls in boxes and quantum gravity", (September, 1997), [Online Los Alamos Archive Preprint]: cited on 19 November 1998, http://xxx.lanl.gov/abs/hep-lat/9709056. 4.6

[48] Bialas, P., Burda, Z., Krzywicki, A., and Petersson, B., "Focusing on the fixed point of 4-d simplicial gravity", Nucl. Phys. B, 472, 293-308, (1996). For a related online version see: P. Bialas, et al., "Focusing on the fixed point of 4-d simplicial gravity", (January, 1996), [Online Los Alamos Archive Preprint]: cited on 19 November 1998, http://xxx.lanl.gov/ $\mathrm{abs} / \mathrm{hep}-\mathrm{lat} / 9601024 . \quad 4.5,4.6,4.12,4.14$ 
[49] Bialas, P., Burda, Z., Petersson, B., and Tabaczek, J., "Appearance of mother universe and singular vertices in random geometries", Nucl. Phys. $B, 495,463-476$, (1997). For a related online version see: P. Bialas, et al., "Appearance of mother universe and singular vertices in random geometries", (August, 1996), [Online Los Alamos Archive Preprint]: cited on 19 November 1998, http://xxx.lanl.gov/abs/hep-lat/9608030. 4.6, 4.11

[50] Bilke, S., Burda, Z., and Jurkiewicz, J., "Simplicial quantum gravity on a computer", Computer Phys. Commun., 85, 278-292, (1995). For a related online version see: S. Bilke, et al., "Simplicial quantum gravity on a computer", (March, 1994), [Online Los Alamos Archive Preprint]: cited on 19 November 1998, http://xxx.lanl.gov/abs/hep-lat/9403017. 4.4

[51] Bilke, S., Burda, Z., Krzywicki, A., and Petersson, B., "Phase transition and topology in 4-d simplicial gravity", Nucl. Phys. Proc. Suppl., 53, 743-745, (1997). For a related online version see: S. Bilke, et al., "Phase transition and topology in 4-d simplicial gravity", (August, 1996), [Online Los Alamos Archive Preprint]: cited on 19 November 1998, http://xxx. lanl.gov/abs/hep-lat/9608027. 4.4, 4.6, 4.10

[52] Bilke, S., Burda, Z., Krzywicki, A., Petersson, B., Tabaczek, J., and Thorleifsson, G., "4-d simplicial quantum gravity interacting with gauge matter fields", Phys. Lett. B, 418, 266-272, (1998). For a related online version see: S. Bilke, et al., "4-d simplicial quantum gravity interacting with gauge matter fields", (October, 1997), [Online Los Alamos Archive Preprint]: cited on 19 November 1998, http://xxx.lanl.gov/ abs/hep-lat/9710077. 4.9

[53] Bilke, S., Burda, Z., and Petersson, B., "Topology in 4-d simplicial quantum gravity", Phys. Lett. B, 395, 4-9, (1997). For a related online version see: S. Bilke, et al., "Topology in 4-d simplicial quantum gravity", (November, 1996), [Online Los Alamos Archive Preprint]: cited on 19 November 1998, http://xxx.lanl.gov/abs/hep-lat/9611020. 4.4, 4.10

[54] Bowick, M., "Random surfaces and lattice gravity", Nucl. Phys. Proc. Suppl., 63, 77-88, (1998). For a related online version see: M. Bowick, "Random surfaces and lattice gravity", (October, 1997), [Online Los Alamos Archive Preprint]: cited on 19 November 1998, http: //xxx.lanl.gov/abs/hep-lat/9710005. 4.1

[55] Brewin, L., "The Riemann and extrinsic curvature tensors in the Regge calculus", Class. Quantum Grav., 5, 1193-1203, (1988). 3.2

[56] Brügmann, B., "Measure of four-dimensional simplicial quantum gravity", Nucl. Phys. Proc. Suppl., 30, 760-763, (1993). 4.7 
[57] Brügmann, B., "Nonuniform measure in four-dimensional simplicial quantum gravity", Phys. Rev. D, 47, 3330-3338, (1993). For a related online version see: B. Brügmann, "Nonuniform measure in four-dimensional simplicial quantum gravity", (October, 1992), [Online Los Alamos Archive Preprint]: cited on 19 November 1998, http://xxx.lanl.gov/abs/ hep-lat/9210001. 4.4, 4.7

[58] Brügmann, B., and Marinari, E., "4-d simplicial quantum gravity with a nontrivial measure", Phys. Rev. Lett., 70, 1908-1911, (1993). For a related online version see: B. Brügmann, et al., "4-d simplicial quantum gravity with a nontrivial measure", (October, 1992), [Online Los Alamos Archive Preprint]: cited on 19 November 1998, http://xxx.lanl.gov/ abs/hep-lat/9210002. 4.7

[59] Brügmann, B., and Marinari, E., "Monte Carlo simulations of 4d simplicial quantum gravity", J. Math. Phys., 36, 6340-6352, (1995). For a related online version see: B. Brügmann, et al., "Monte Carlo simulations of 4d simplicial quantum gravity", (April, 1995), [Online Los Alamos Archive Preprint]: cited on 19 November 1998, http://xxx.lanl.gov/ abs/hep-lat/9504004. 4.1

[60] Brügmann, B., and Marinari, E., "More on the exponential bound of four-dimensional simplicial quantum gravity", Phys. Lett. B, 349, 35-41, (1995). For a related online version see: B. Brügmann, et al., "More on the exponential bound of four-dimensional simplicial quantum gravity", (November, 1994), [Online Los Alamos Archive Preprint]: cited on 19 November 1998, http://xxx.lanl.gov/abs/hep-th/9411060. 4.3

[61] Burda, Z., Kownacki, J.P., and Krzywicki, A., "Towards a nonperturbative renormalization of Euclidean quantum gravity", Phys. Lett. B, 356, 466-471, (1995). For a related online version see: Z. Burda, et al., "Towards a nonperturbative renormalization of Euclidean quantum gravity", (May, 1995), [Online Los Alamos Archive Preprint]: cited on 19 November 1998, http://xxx.lanl.gov/abs/hep-th/9505104. 4.12

[62] Caracciolo, S., Menotti, P., and Pelissetto, A., "Lattice supergravity and graviton-gravitino doubling", Nucl. Phys. B, 296, 868-876, (1988). 2.5

[63] Caracciolo, S., and Pelissetto, A., "Analysis of the critical behavior or the de Sitter quantum gravity on a hypercubic lattice", Phys. Lett. B, 193, 237-240, (1987). 2.3

[64] Caracciolo, S., and Pelissetto, A., "Nonperturbative lattice gravity", Nucl. Phys. Proc. Suppl., 4, 78-82, (1987). 2.3

[65] Caracciolo, S., and Pelissetto, A., "A numerical investigation about quantum measure in lattice gravity", Phys. Lett. B, 207, 468-470, (1988). 2.3 
[66] Caracciolo, S., and Pelissetto, A., "Phases and topological structures of de Sitter lattice gravity", Nucl. Phys. B, 299, 693-718, (1988). 2.3

[67] Caracciolo, S., and Pelissetto, A., "From lattice gauge theory towards gravity", in Damgaard, P.H. et al., ed., Probabilistic methods in quantum field theory and quantum gravity, 37-54, (Plenum Press, New York, 1990). 2.3

[68] Carfora, M., and Marzuoli, A., "Entropy estimates for simplicial quantum gravity", J. Geom. Phys., 16, 99-119, (1995). 4.3

[69] Caselle, M., D'Adda, A., and Magnea, L., "Doubling of all matter fields coupled with gravity on a lattice", Phys. Lett. B, 192, 411-414, (1987). 2.7

[70] Caselle, M., D'Adda, A., and Magnea, L., "Lattice gravity and supergravity as spontaneously broken gauge theories of the (super)Poincaré group", Phys. Lett. B, 192, 406-410, (1987). 2.7

[71] Caselle, M., D'Adda, A., and Magnea, L., "Regge Calculus as a local theory of the Poincaré group", Phys. Lett. B, 232, 457-461, (1989). 3.9, 3.14

[72] Catterall, S., "Simulations of dynamically triangulated gravity", Computer Phys. Commun., 87, 409-415, (1995). For a related online version see: S. Catterall, "Simulations of dynamically triangulated gravity", (May, 1994), [Online Los Alamos Archive Preprint]: cited on 19 November 1998, http://xxx.lanl.gov/abs/hep-lat/9405026. 4.4

[73] Catterall, S., "Lattice quantum gravity: review and recent developments", Nucl. Phys. Proc. Suppl., 47, 59-70, (1996). For a related online version see: S. Catterall, "Lattice quantum gravity: review and recent developments", (October, 1995), [Online Los Alamos Archive Preprint]: cited on 19 November 1998, http://xxx.lanl.gov/abs/hep-lat/9510008. 4.1

[74] Catterall, S., Kogut, J., and Renken, R., "Is there an exponential bound in four-dimensional simplicial gravity?", Phys. Rev. Lett., 72, 4062-4065, (1994). For a related online version see: S. Catterall, et al., "On the absence of an exponential bound in four dimensional simplicial gravity", (March, 1994), [Online Los Alamos Archive Preprint]: cited on 19 November 1998, http://xxx.lanl.gov/abs/hep-lat/9403019. 4.3

[75] Catterall, S., Kogut, J., and Renken, R., "Phase structure of fourdimensional simplicial quantum gravity", Phys. Lett. B, 328, 277-283, (1994). For a related online version see: S. Catterall, et al., "Phase structure of four-dimensional simplicial quantum gravity", (January, 1994), [Online Los Alamos Archive Preprint]: cited on 19 November 1998, http://xxx.lanl.gov/abs/hep-lat/9401026. 4.5, 4.6, 4.13 
[76] Catterall, S., Kogut, J., and Renken, R., "Simulations of four-dimensional simplicial quantum gravity", Nucl. Phys. Proc. Suppl., 34, 733-735, (1994). $\quad 4.5,4.6,4.13$

[77] Catterall, S., Kogut, J., and Renken, R., "Singular structure in 4-d simplicial gravity", Phys. Lett. B, 416, 274-280, (1998). For a related online version see: S. Catterall, et al., "Singular structure in 4-d simplicial gravity", (September, 1997), [Online Los Alamos Archive Preprint]: cited on 19 November 1998, http://xxx.lanl.gov/abs/hep-lat/9709007. 4.5, $4.6,4.11$

[78] Catterall, S., Kogut, J., Renken, R., and Thorleifsson, G., "Baby universes in 4-d dynamical triangulation", Phys. Lett. B, 366, 72-76, (1996). For a related online version see: S. Catterall, et al., "Baby universes in 4-d dynamical triangulation", (September, 1995), [Online Los Alamos Archive Preprint]: cited on 19 November 1998, http://xxx.lanl.gov/ abs/hep-lat/9509004. 4.3

[79] Catterall, S., Thorleifsson, G., Kogut, J., and Renken, R., "Singular vertices and the triangulation space of the d sphere", Nucl. Phys. B, 468, 263-276, (1996). For a related online version see: S. Catterall, et al., "Singular vertices and the triangulation space of the d sphere", (December, 1995), [Online Los Alamos Archive Preprint]: cited on 19 November 1998, http://xxx.lanl.gov/abs/hep-lat/9512012. 4.4, 4.11

[80] Catterall, S., Thorleifsson, G., Kogut, J., and Renken, R., "Simplicial gravity in dimension greater than two", Nucl. Phys. Proc. Suppl., 53, 756-759, (1997). For a related online version see: S. Catterall, et al., "Simplicial gravity in dimension greater than two", (August, 1996), [Online Los Alamos Archive Preprint]: cited on 19 November 1998, http://xxx.lanl.gov/abs/hep-lat/9608042. 4.11

[81] Cheeger, J., Müller, W., and Schrader, R., "Lattice gravity or Riemannian structure on piecewise linear spaces", in Breitenlohner, P., and Dürr, H.P., eds., Unified theories of elementary particles, volume 160 of Lecture Notes in Physics, 176-188, (Springer, Berlin, 1982). 3.1, 3.1, 3.2, 3.13

[82] Cheeger, J., Müller, W., and Schrader, R., "On the curvature of piecewise flat spaces", Commun. Math. Phys., 92, 405-454, (1984). 3.2, 3.7

[83] Corichi, A., and Zapata, J.A., "On diffeomorphism invariance for lattice theories", Nucl. Phys. B, 493, 475-490, (1997). For a related online version see: A. Corichi, et al., "On diffeomorphism invariance for lattice theories", (November, 1996), [Online Los Alamos Archive Preprint]: cited on 19 November 1998, http://xxx.lanl.gov/abs/gr-qc/9611034. 2.14

[84] Das, A., Kaku, M., and Townsend, P.K., "Lattice formulation of general relativity", Phys. Lett. B, 81, 11-14, (1979). 2.4 
[85] David, F., "Simplicial quantum gravity and random lattices", in Julia, B., and Zinn-Justin, J., eds., Gravitation and quantizations (Les Houches Summer School, Session LVII, 1992), 679-750, (Elsevier, Amsterdam, 1995). For a related online version see: F. David, "Simplicial quantum gravity and random lattices", (March, 1993), [Online Los Alamos Archive Preprint]: cited on 19 November 1998, http://xxx.lanl.gov/abs/ hep-th/9303127. 4.1

[86] de Bakker, B.V., "Absence of barriers in dynamical triangulation", Phys. Lett. B, 348, 35-38, (1995). For a related online version see: B.V. de Bakker, "Absence of barriers in dynamical triangulation", (November, 1994), [Online Los Alamos Archive Preprint]: cited on 19 November 1998, http://xxx.lanl.gov/abs/hep-lat/9411070. 4.4

[87] de Bakker, B.V., "Further evidence that the transition of 4-d dynamical triangulation is first order", Phys. Lett. B, 389, 238-242, (1996). For a related online version see: B.V. de Bakker, "Further evidence that the transition of 4-d dynamical triangulation is first order", (March, 1996), [Online Los Alamos Archive Preprint]: cited on 19 November 1998, http: //xxx.lanl.gov/abs/hep-lat/9603024. 4.6

[88] de Bakker, B.V., and Smit, J., "Euclidean gravity attracts", Nucl. Phys. Proc. Suppl., 34, 739-741, (1994). For a related online version see: B.V. de Bakker, et al., "Euclidean gravity attracts", (November, 1993), [Online Los Alamos Archive Preprint]: cited on 19 November 1998, http://xxx. lanl.gov/abs/hep-lat/9311064. 4.13

[89] de Bakker, B.V., and Smit, J., "Volume dependence of the phase boundary in 4-d dynamical riangulation", Phys. Lett. B, 334, 304-308, (1994). For a related online version see: B.V. de Bakker, et al., "Volume dependence of the phase boundary in 4-d dynamical triangulation", (May, 1994), [Online Los Alamos Archive Preprint]: cited on 19 November 1998, http://xxx. lanl.gov/abs/hep-lat/9405013. 4.3, 4.5, 4.8

[90] de Bakker, B.V., and Smit, J., "Curvature and scaling in 4-d dynamical triangulation", Nucl. Phys. B, 439, 239-258, (1995). For a related online version see: B.V. de Bakker, et al., "Curvature and scaling in 4d dynamical triangulation", (July, 1994), [Online Los Alamos Archive Preprint]: cited on 19 November 1998, http://xxx.lanl.gov/abs/ hep-lat/9407014. 4.5, 4.6, 4.13

[91] de Bakker, B.V., and Smit, J., "Two point functions in 4-d dynamical triangulation", Nucl. Phys. B, 454, 343-356, (1995). For a related online version see: B.V. de Bakker, et al., "Two point functions in 4-d dynamical triangulation", (March, 1995), [Online Los Alamos Archive Preprint]: cited on 19 November 1998, http://xxx.lanl.gov/abs/hep-lat/9503004. $4.6,4.14$ 
[92] de Bakker, B.V., and Smit, J., "Correlations and binding in 4-d dynamical triangulation", Nucl. Phys. Proc. Suppl., 47, 613-616, (1996). For a related online version see: B.V. de Bakker, et al., "Correlations and binding in 4-d dynamical triangulation", (October, 1995), [Online Los Alamos Archive Preprint]: cited on 19 November 1998, http: //xxx.lanl.gov/abs/hep-lat/9510041. 4.6, 4.13, 4.14

[93] de Bakker, B.V., and Smit, J., "Gravitational binding in 4-d dynamical triangulation", Nucl. Phys. B, 484, 476-494, (1997). For a related online version see: B.V. de Bakker, et al., "Gravitational binding in 4-d dynamical triangulation", (April, 1996), [Online Los Alamos Archive Preprint]: cited on 19 November 1998, http://xxx.lanl.gov/abs/hep-lat/9604023. 4.13

[94] De Pietri, R., and Rovelli, C., "Geometry eigenvalues and scalar product from recoupling theory in loop quantum gravity", Phys. Rev. D, 54, 26642690, (1996). For a related online version see: R. De Pietri, et al., "Geometry eigenvalues and scalar product from recoupling theory in loop quantum gravity", (February, 1996), [Online Los Alamos Archive Preprint]: cited on 19 November 1998, http://xxx.lanl.gov/abs/gr-qc/9602023. 2.15

[95] Egawa, H.S., Hotta, T., Izubuchi, T., Tsuda, N., and Yukawa, T., "Scaling behaviour in 4-d simplicial quantum gravity", Prog. Theor. Phys., 97, 539-552, (1997). For a related online version see: H.S. Egawa, et al., "Scaling behaviour in 4-d simplicial quantum gravity", (November, 1996), [Online Los Alamos Archive Preprint]: cited on 19 November 1998, http: //xxx.lanl.gov/abs/hep-lat/9611028. 4.6

[96] Egawa, H.S., Hotta, T., Izubuchi, T., Tsuda, N., and Yukawa, T., "Scaling structures in four-dimensional simplicial gravity", Nucl. Phys. Proc. Suppl., 53, 760-762, (1997). For a related online version see: H.S. Egawa, et al., "Scaling structures in four-dimensional simplicial gravity", (August, 1996), [Online Los Alamos Archive Preprint]: cited on 19 November 1998, http://xxx.lanl.gov/abs/hep-lat/9608149. 4.6

[97] Egawa, H.S., Tsuda, N., and Yukawa, T., "Common structures in 2-d, 3-d and 4-d simplicial quantum gravity", Nucl. Phys. Proc. Suppl., 63, 736738 , (1998). For a related online version see: H.S. Egawa, et al., "Common structures in 2-d, 3-d and 4-d simplicial quantum gravity", (September, 1997), [Online Los Alamos Archive Preprint]: cited on 19 November 1998, http://xxx.lanl.gov/abs/hep-lat/9709099. 4.4

[98] Egawa, H.S., Tsuda, N., and Yukawa, T., "Common structures in simplicial quantum gravity", preprint KEK-TH-561, 10pp, (1998). 4.4

[99] Ezawa, K., "Multi-plaquette solutions for discretized Ashtekar gravity", Mod. Phys. Lett. A, 11, 2921-2932, (1996). 2.11, 2.13 
[100] Ezawa, K., "Nonperturbative solutions for canonical quantum gravity: an overview", Phys. Rep., 286, 271-348, (1997). For a related online version see: K. Ezawa, "Nonperturbative solutions for canonical quantum gravity: an overview", (January, 1996), [Online Los Alamos Archive Preprint]: cited on 19 November 1998, http://xxx.lanl.gov/abs/ gr-qc/9601050. 2.13

[101] Feinberg, G., Friedberg, R., Lee, T.D., and Ren, H.C., "Lattice gravity near the continuum limit", Nucl. Phys. B, 245, 343-368, (1984). 3.1, 3.2

[102] Fort, H., Gambini, R., and Pullin, J., "Lattice knot theory and quantum gravity in the loop representation", Phys. Rev. D, 56, 2127-2143, (1997). For a related online version see: H. Fort, et al., "Lattice knot theory and quantum gravity in the loop representation", (August, 1996), [Online Los Alamos Archive Preprint]: cited on 19 November 1998, http://xxx. lanl.gov/abs/gr-qc/9608033. 2.13

[103] Friedberg, R., and Lee, T.D., "Derivation of Regge's action from Einstein's theory of general relativity", Nucl. Phys. B, 242, 145-166, (1984). 3.2

[104] Fröhlich, J., "Regge calculus and discretized gravitational functional integrals", in Fröhlich, J., ed., Non-perturbative quantum field theory: Mathematical aspects and applications. Selected papers., 523-545, (World Scientific, Singapore, 1992). unpublished preprint IHES, 1981. 3.1, 3.1, 3.2, $3.9,3.14$

[105] Gross, M., and Varsted, S., "Elementary moves and ergodicity in ddimensional simplicial quantum gravity", Nucl. Phys. B, 378, 367-380, (1992). 4.4

[106] Hamber, H.W., "Simplicial quantum gravity", in Osterwalder, K., and Stora, R., eds., Critical phenomena, random systems, gauge theories (Les Houches Summer School, Session XLIII, 1984), 375-439, (Elsevier, Amsterdam, 1986). $\quad 3.1,3.2,3.3$

[107] Hamber, H.W., "Simplicial quantum gravity from two-dimensions to fourdimensions", in Damgaard, P.H. et al., ed., Probabilistic methods in quantum field theory and quantum gravity, 77-87, (Plenum Press, New York, 1990). $\quad 3.1$

[108] Hamber, H.W., "Critical behavior in simplicial quantum gravity", Nucl. Phys. Proc. Suppl., 20, 728-732, (1991). 3.4

[109] Hamber, H.W., "Simulations of discrete quantized gravity", Int. J. Supercomput. Appl., 5, 84-97, (1991). 3.4

[110] Hamber, H.W., "Fluctuations and correlations in simplicial quantum gravity", Nucl. Phys. Proc. Suppl., 26, 581-583, (1992). 3.1 
[111] Hamber, H.W., "Phases of 4-d simplicial quantum gravity", Phys. Rev. $D, \mathbf{4 5}, 507-512,(1992)$. 3.4, 3.6, 3.15

[112] Hamber, H.W., "Phases of simplicial quantum gravity", Nucl. Phys. Proc. Suppl., 25, 150-175, (1992). 3.1

[113] Hamber, H.W., "Phases of simplicial quantum gravity in four dimensions: estimates for the critical exponents", Nucl. Phys. B, 400, 347-389, (1993). $3.6,3.15$

[114] Hamber, H.W., "Smooth and rough phases of quantized gravity", Nucl. Phys. Proc. Suppl., 30, 751-755, (1993). 3.4

[115] Hamber, H.W., "Invariant correlations in simplicial gravity", Phys. Rev. $D$, 50, 3932-3941, (1994). For a related online version see: H.W. Hamber, "Invariant correlations in simplicial gravity", (November, 1993), [Online Los Alamos Archive Preprint]: cited on 19 November 1998, http://xxx. lanl.gov/abs/hep-th/9311024. 3.8

[116] Hamber, H.W., "Scalar fields coupled to four-dimensional lattice gravity", in Carr, J., and Perrottet, M., eds., High energy physics: 1993 International Europhysics Conference, 211-213, (Éditions Frontières, Gif-sur-Yvette, 1994). For a related online version see: H.W. Hamber, "Scalar fields coupled to four-dimensional lattice gravity", (October, 1993), [Online Los Alamos Archive Preprint]: cited on 19 November 1998, http://xxx.lanl.gov/abs/hep-th/9310152. 3.1, 3.11

[117] Hamber, H.W., and Williams, R.M., "Higher derivative quantum gravity on a simplicial lattice", Nucl. Phys. B, 248, 392-415, (1984). 3.2

[118] Hamber, H.W., and Williams, R.M., "Nonperturbative simplicial quantum gravity", Phys. Lett. B, 157, 368-374, (1985). 3.3

[119] Hamber, H.W., and Williams, R.M., "Simplicial quantum gravity with higher derivative terms: formalism and numerical results in fourdimensions", Nucl. Phys. B, 269, 712-734, (1986). 3.2, 3.2, 3.3

[120] Hamber, H.W., and Williams, R.M., "Simplicial gravity coupled to scalar matter", Nucl. Phys. B, 415, 463-496, (1994). For a related online version see: H.W. Hamber, et al., "Simplicial gravity coupled to scalar matter", (August, 1993), [Online Los Alamos Archive Preprint]: cited on 19 November 1998, http://xxx.lanl.gov/abs/hep-th/9308099. 3.11

[121] Hamber, H.W., and Williams, R.M., "Newtonian potential in quantum Regge gravity", Nucl. Phys. B, 435, 361-398, (1995). For a related online version see: H.W. Hamber, et al., "Newtonian potential in quantum Regge gravity", (June, 1994), [Online Los Alamos Archive Preprint]: cited on 19 November 1998, http://xxx.lanl.gov/abs/hep-th/9406163. 3.12 
[122] Hamber, H.W., and Williams, R.M., "Gauge invariance in simplicial gravity", Nucl. Phys. B, 487, 345-408, (1997). For a related online version see: H.W. Hamber, et al., "Gauge invariance in simplicial gravity", (July, 1996), [Online Los Alamos Archive Preprint]: cited on 19 November 1998, http://xxx.lanl.gov/abs/hep-th/9607153. 3.13

[123] Hamber, H.W., and Williams, R.M., "On the measure in simplicial gravity", preprint DAMTP-97-75, 15pp, (1997). 3.13

[124] Hartle, J.B., "Simplicial minisuperspace. I. General discussion", J. Math. Phys., 26, 804-814, (1985). 3.1, 3.13, 3.14

[125] Hartle, J.B., "Numerical quantum gravity", in Sato, H., and Nakamura, T., eds., Proceedings of the 14th Yamada conference on gravitational collapse and relativity, 329-338, (World Scientific, Singapore, 1986). 3.14

[126] Hartle, J.B., "Simplicial minisuperspace. II. Some classical solutions on simple triangulations", J. Math. Phys., 27, 287-295, (1986). 3.14

[127] Hartle, J.B., "Simplicial minisuperspace. III. Integration contours in a five-simplex model", J. Math. Phys., 30, 452-460, (1989). 3.14

[128] Hartle, J.B., and Sorkin, R., "Boundary terms in the action for the Regge calculus", Gen. Relativ. Gravit., 13, 541-549, (1981). 3.1

[129] Hotta, T., Izubuchi, T., and Nishimura, J., "Singular vertices in the strong coupling phase of four-dimensional simplicial gravity", Prog. Theor. Phys., 94, 263-270, (1995). For a related online version see: T. Hotta, et al., "Singular vertices in the strong coupling phase of four-dimensional simplicial gravity", (September, 1997), [Online Los Alamos Archive Preprint]: cited on 19 November 1998, http://xxx.lanl.gov/abs/hep-lat/9709073. $4.5,4.11$

[130] Hotta, T., Izubuchi, T., and Nishimura, J., "Singular vertices in the strong coupling phase of four-dimensional simplicial gravity", Nucl. Phys. Proc. Suppl., 47, 609-612, (1996). For a related online version see: T. Hotta, et al., "Singular vertices in the strong coupling phase of fourdimensional simplicial gravity", (November, 1995), [Online Los Alamos Archive Preprint]: cited on 19 November 1998, http://xxx.lanl.gov/ abs/hep-lat/9511023. 4.11

[131] Immirzi, G., "Quantizing Regge calculus", Class. Quantum Grav., 13, 2385-2394, (1996). For a related online version see: G. Immirzi, "Quantizing Regge calculus", (December, 1995), [Online Los Alamos Archive Preprint]: cited on 19 November 1998, http://xxx.lanl.gov/abs/ gr-qc/9512040. 3.14 
[132] Immirzi, G., "Quantum gravity and Regge calculus", Nucl. Phys. Proc. Suppl., 57, 65-72, (1997). For a related online version see: G. Immirzi, "Quantum gravity and Regge calculus", (January, 1997), [Online Los Alamos Archive Preprint]: cited on 19 November 1998, http: //xxx.lanl.gov/abs/gr-qc/9701052. 3.14

[133] Isham, C.J., "Quantum gravity", in MacCallum, M.A.H., ed., Proceedings of the 11th International Conference on General Relativity and Gravitation, 99-127, (Cambridge University Press, Cambridge, 1987). 3.1

[134] Jevicki, A., and Ninomiya, M., "Functional formulation of Regge gravity", Phys. Rev. D, 33, 1634-1637, (1986). 3.13

[135] Johnston, D.A., "Gravity and random surfaces on the lattice: a review", Nucl. Phys. Proc. Suppl., 53, 43-55, (1997). For a related online version see: D.A. Johnston, "Gravity and random surfaces on the lattice: a review", (July, 1996), [Online Los Alamos Archive Preprint]: cited on 19 November 1998, http://xxx.lanl.gov/abs/hep-lat/9607021. 4.1

[136] Jurkiewicz, J., "Simplicial gravity and random surfaces", Nucl. Phys. Proc. Suppl., 30, 108-121, (1993). 4.1

[137] Kaku, M., "Lattices, gravity and supergravity", in Hawking, S.W., and Roček, M., eds., Superspace and supergravity, 503-515, (Cambridge University Press, Cambridge, 1981). 2.4

[138] Kaku, M., "Strong-coupling approach to the quantization of conformal gravity", Phys. Rev. D, 27, 2819-2834, (1983). 2.4

[139] Kaku, M., "Generally covariant lattices, the random calculus, and the strong coupling approach to the renormalization of gravity", in Batalin, I.A. et al., ed., Quantum field theory and quantum statistics, vol. 2, 141163, (Hilger, Bristol, 1987). 2.4

[140] Kawamoto, N., and Nielsen, H.B., "Lattice gauge gravity with fermions", Phys. Rev. D, 43, 1150-1156, (1991). 3.9, 3.14

[141] Khatsymovsky, V., "On the quantization of Regge links", Phys. Lett. B, 323, 292-295, (1994). 3.14

[142] Kondo, K., "Euclidean quantum gravity on a flat lattice", Prog. Theor. Phys., 72, 841-852, (1984). 2.4

[143] Kristjansen, C.F., "Higher derivative regularization in 4-d quantum gravity", Nucl. Phys. Proc. Suppl., 30, 756-759, (1993). 4.8

[144] Krzywicki, A., "Perspectives in lattice gravity", Acta Phys. Pol. B, 27, 827-838, (1996). For a related online version see: A. Krzywicki, "Perspectives in lattice gravity", (December, 1995), [Online Los Alamos Archive Preprint]: cited on 19 November 1998, http://xxx.lanl.gov/abs/ hep-lat/9512023. 4.1

Living Reviews in Relativity (1998-13)

http://www.livingreviews.org 
[145] Leutwyler, H., "Gravitational field: equivalence of Feynman quantization and canonical quantization", Phys. Rev., 134, 1155-1182, (1964). 1

[146] Loll, R., "Non-perturbative solutions for lattice quantum gravity", Nucl. Phys. B, 444, 619-639, (1995). For a related online version see: R. Loll, "Non-perturbative solutions for lattice quantum gravity", (February, 1995), [Online Los Alamos Archive Preprint]: cited on 19 November 1998, http://xxx.lanl.gov/abs/gr-qc/9502006. 2.11, 2.13, 2.14

[147] Loll, R., "The volume operator in discretized quantum gravity", Phys. Rev. Lett., 75, 3048-3051, (1995). For a related online version see: R. Loll, "The volume operator in discretized quantum gravity", (June, 1995), [Online Los Alamos Archive Preprint]: cited on 19 November 1998, http://xxx.lanl.gov/abs/gr-qc/9506014. 2.15

[148] Loll, R., "A real alternative to quantum gravity in loop space", Phys. Rev. D, 54, 5381-5384, (1996). For a related online version see: R. Loll, "A real alternative to quantum gravity in loop space", (February, 1996), [Online Los Alamos Archive Preprint]: cited on 19 November 1998, http: //xxx.lanl.gov/abs/gr-qc/9602041. 2.16

[149] Loll, R., "Spectrum of the volume operator in quantum gravity", Nucl. Phys. B, 460, 143-154, (1996). For a related online version see: R. Loll, "Spectrum of the volume operator in quantum gravity", (November, 1995), [Online Los Alamos Archive Preprint]: cited on 19 November 1998, http://xxx.lanl.gov/abs/gr-qc/9511030. 2.15

[150] Loll, R., "Further results on geometric operators in quantum gravity", Class. Quantum Grav., 14, 1725-1741, (1997). For a related online version see: R. Loll, "Further results on geometric operators in quantum gravity", (December, 1996), [Online Los Alamos Archive Preprint]: cited on 19 November 1998, http://xxx.lanl.gov/abs/gr-qc/9612068. 2.15

[151] Loll, R., "Imposing det E > 0 in discrete quantum gravity", Phys. Lett. $B$, 399, 227-232, (1997). For a related online version see: R. Loll, "Imposing det E $>0$ in discrete quantum gravity", (March, 1997), [Online Los Alamos Archive Preprint]: cited on 19 November 1998, http: //xxx.lanl.gov/abs/gr-qc/9703033. 2.15

[152] Loll, R., "Latticing quantum gravity", Nucl. Phys. Proc. Suppl., 57, 255258, (1997). For a related online version see: R. Loll, "Latticing quantum gravity", (January, 1997), [Online Los Alamos Archive Preprint]: cited on 19 November 1998, http://xxx.lanl.gov/abs/gr-qc/9701007. 2.15

[153] Loll, R., "Quantizing canonical gravity in the real domain", in Dremin, I.M., and Semikhatov, A.M., eds., Proceedings of the 2nd International Sakharov Conference on Physics, 280-283, (World Scientific, Singapore, 1997). For a related online version see: R. Loll, "Quantizing canonical gravity in the real domain", (January, 1997), [Online Los Alamos 
Archive Preprint]: cited on 19 November 1998, http://xxx.lanl.gov/ abs/gr-qc/9701031. 2.16

[154] Loll, R., "Simplifying the spectral analysis of the volume operator", Nucl. Phys. B, 500, 405-420, (1997). For a related online version see: R. Loll, "Simplifying the spectral analysis of the volume operator", (June, 1997), [Online Los Alamos Archive Preprint]: cited on 19 November 1998, http: //xxx.lanl.gov/abs/gr-qc/9706038. 2.15

[155] Loll, R., "Still on the way to quantizing gravity", in Bassan, M. et al., ed., Proceedings of the 12th Italian Conference on General Relativity and Gravitational Physics, 280-283, (World Scientific, Singapore, 1997). For a related online version see: R. Loll, "Still on the way to quantizing gravity", (January, 1997), [Online Los Alamos Archive Preprint]: cited on 19 November 1998, http://xxx.lanl.gov/abs/gr-qc/9701032. 2.10

[156] Loll, R., "On the diffeomorphism commutators of lattice quantum gravity", Class. Quantum Grav., 15, 799-809, (1998). For a related online version see: R. Loll, "On the diffeomorphism commutators of lattice quantum gravity", (August, 1997), [Online Los Alamos Archive Preprint]: cited on 19 November 1998, http://xxx.lanl.gov/abs/gr-qc/9708025. 2.12

[157] MacDowell, S.W., and Mansouri, R., "Unified geometric theory of gravity and supergravity", Phys. Rev. Lett., 38, 739-742, (1977). 2.2

[158] Mäkelä, J., "Phase space coordinates and the Hamiltonian constraint of Regge calculus", Phys. Rev. D, 49, 2882-2896, (1994). 3.14

[159] Mannion, C.L.T., and Taylor, J.G., "General relativity on a flat lattice", Phys. Lett. B, 100, 261-266, (1981). 2.4

[160] Menotti, P., "Nonperturbative quantum gravity", Nucl. Phys. Proc. Suppl., 17, 29-38, (1990). 3.1

[161] Menotti, P., "The role of diffeomorphisms in the integration over a finite dimensional space of geometries", Nucl. Phys. Proc. Suppl., 63, 760-762, (1998). For a related online version see: P. Menotti, "The role of diffeomorphisms in the integration over a finite dimensional space of geometries", (September, 1997), [Online Los Alamos Archive Preprint]: cited on 19 November 1998, http://xxx.lanl.gov/abs/hep-lat/9709101. 3.13

[162] Menotti, P., and Peirano, P.P., "Diffeomorphism invariant measure for finite dimensional geometries", Nucl. Phys. B, 488, 719-734, (1997). For a related online version see: P. Menotti, et al., "Diffeomorphism invariant measure for finite dimensional geometries", (July, 1996), [Online Los Alamos Archive Preprint]: cited on 19 November 1998, http: //xxx.lanl.gov/abs/hep-th/9607071. 3.13 
[163] Menotti, P., and Peirano, P.P., "Functional integration for Regge gravity", Nucl. Phys. Proc. Suppl., 57, 82-90, (1997). For a related online version see: P. Menotti, et al., "Functional integration for Regge gravity", (February, 1997), [Online Los Alamos Archive Preprint]: cited on 19 November 1998, http://xxx.lanl.gov/abs/gr-qc/9702020. 3.13

[164] Menotti, P., and Peirano, P.P., "Functional integration on Regge geometries", Nucl. Phys. Proc. Suppl., 53, 780-782, (1997). For a related online version see: P. Menotti, et al., "Functional integration on Regge geometries", (July, 1996), [Online Los Alamos Archive Preprint]: cited on 19 November 1998, http://xxx.lanl.gov/abs/hep-lat/9607073. 3.13

[165] Menotti, P., and Pelissetto, A., "Reflection positivity and graviton doubling in Euclidean lattice gravity", Annals Phys., 170(2), 287-309, (1986). 2.5

[166] Menotti, P., and Pelissetto, A., "Gauge invariance and functional integration measure in lattice gravity", Nucl. Phys. B, 288, 813-831, (1987). 2.6

[167] Menotti, P., and Pelissetto, A., "Poincaré, de Sitter, and conformal gravity on the lattice", Phys. Rev. D, 35, 1194-1204, (1987). 2.5

[168] Misner, C.W., Thorne, K.S., and Wheeler, J.A., Gravitation, (Freeman, San Francisco, 1973). 3.1

[169] Myers, E., "Unbounded action and 'density of states"', Class. Quantum Grav., 9, 405-411, (1992). 3.3

[170] Nabutovsky, A., and Ben-Av, R., "Noncomputability arising in dynamical triangulation model of four-dimensional quantum gravity", Commun. Math. Phys., 157, 93-98, (1993). 4.4

[171] Pachner, U., "Konstruktionsmethoden und das kombinatorische Homöomorphieproblem für Triangulationen kompakter semilinearer Mannigfaltigkeiten", Abh. Math. Sem. Univ. Hamburg, 57, 69-85, (1986). 4.4

[172] Pachner, U., "P.L. homeomorphic manifolds are equivalent by elementary shellings", Europ. J. Combinatorics, 12, 129-145, (1991). 4.4

[173] Rebbi, C., Lattice gauge theories and Monte-Carlo simulations, (World Scientific, Singapore, 1983). 2.1

[174] Regge, T., "General Relativity without coordinates", Nuovo Cimento A, 19, 558-571, (1961). $\quad 3.1,3.2$

[175] Reisenberger, M.P., "A lattice worldsheet sum for 4-d Euclidean general relativity", preprint Montevideo U., 51pp, (1997). 2.7

[176] Ren, H.-C., "Matter fields in lattice gravity", Nucl. Phys. B, 301, 661684, (1988). 3.2 
[177] Renken, R.L., "The renormalization group and dynamical triangulations", Nucl. Phys. Proc. Suppl., 53, 783-785, (1997). For a related online version see: R.L. Renken, "The renormalization group and dynamical triangulations", (October, 1996), [Online Los Alamos Archive Preprint]: cited on 19 November 1998, http://xxx.lanl.gov/abs/hep-lat/9610037. 4.7, 4.12

[178] Renken, R.L., "A renormalization group for dynamical triangulations in arbitrary dimensions", Nucl. Phys. B, 485, 503-516, (1997). For a related online version see: R.L. Renken, "A renormalization group for dynamical triangulations in arbitrary dimensions", (July, 1996), [Online Los Alamos Archive Preprint]: cited on 19 November 1998, http://xxx.lanl.gov/ abs/hep-lat/9607074. 4.7, 4.12

[179] Renteln, P., "Some results of SU(2) spinorial lattice gravity", Class. Quantum Grav., 7, 493-502, (1990). 2.12

[180] Renteln, P., and Smolin, L., "A lattice approach to spinorial quantum gravity", Class. Quantum Grav., 6, 275-294, (1989). 2.10

[181] Roček, M., and Williams, R., "Quantum Regge calculus", Phys. Lett. B, 104, 31-37, (1981). 3.1, 3.1, 3.13

[182] Roček, M., and Williams, R., "Introduction to quantum Regge calculus", in Isham, C., and Duff, M., eds., Quantum structure of space and time, 105-116, (Cambridge University Press, Cambridge, 1982). 3.1

[183] Roček, M., and Williams, R., "The quantization of Regge calculus", Z. Phys. C, 21, 371-381, (1984). 3.1, 3.13

[184] Römer, H., and Zähringer, M., "Functional integration and the diffeomorphism group in Euclidean lattice quantum gravity", Class. Quantum Grav., 3, 897-910, (1986). 3.13, 4.1

[185] Smit, J., "Remarks on the quantum gravity interpretation of 4d dynamical triangulation", Nucl. Phys. Proc. Suppl., 53, 786-790, (1997). For a related online version see: J. Smit, "Remarks on the quantum gravity interpretation of 4d dynamical triangulation", (August, 1996), [Online Los Alamos Archive Preprint]: cited on 19 November 1998, http://xxx.lanl.gov/abs/hep-lat/9608082. 4.13

[186] Smolin, L., "Quantum gravity on a lattice", Nucl. Phys. B, 148, 333-372, (1979). $\quad 2.2$

[187] Sorkin, R., "Time evolution problem in Regge calculus", Phys. Rev. D, 12, 385-396, (1975). $3.1,3.13$

[188] Thiemann, T., "Closed formula for the matrix elements of the volume operator in canonical quantum gravity", J. Math. Phys., 39, 3347-3371, (1998). For a related online version see: T. Thiemann, "Closed formula 
for the matrix elements of the volume operator in canonical quantum gravity", (June, 1996), [Online Los Alamos Archive Preprint]: cited on 19 November 1998, http://xxx.lanl.gov/abs/gr-qc/9606091. 2.15

[189] Tomboulis, E.T., "Unitarity in higher-derivative quantum gravity", Phys. Rev. Lett., 52, 1173-1176, (1984). 2.4

[190] Tuckey, P.A., and Williams, R.M., "Regge calculus: a brief review and bibliography", Class. Quantum Grav., 9, 1409-1422, (1992). 3.1, 3.14

[191] Varsted, S., "Three-dimensional and four-dimensional simplicial quantum gravity", Nucl. Phys. Proc. Suppl., 26, 578-580, (1992). 4.6

[192] Varsted, S., "Four-dimensional quantum gravity by dynamical triangulations", Nucl. Phys. B, 412, 406-414, (1994). 4.5, 4.6

[193] Weingarten, D., "Euclidean quantum gravity on a lattice", Nucl. Phys. B, 210, 229-245, (1982). 4.1

[194] West, P.C., "A geometric gravity Lagrangian", Phys. Lett. B, 76, 569-570, (1978). 2.4

[195] Wheater, J.F., "Random surfaces and lattice quantum gravity", Nucl. Phys. Proc. Suppl., 34, 15-28, (1994). 4.1

[196] Williams, R.M., "Quantum Regge calculus in the Lorentzian domain and its Hamiltonian formulation", Class. Quantum Grav., 3, 853-869, (1986). 3.1

[197] Williams, R.M., "Discrete quantum gravity: the Regge calculus approach", Int. J. Mod. Phys. B, 6, 2097-2108, (1992). 3.1

[198] Williams, R.M., "Simplicial quantum gravity in four dimensions and invariants of discretized manifolds", J. Math. Phys., 36, 6276-6287, (1995). 3.1

[199] Williams, R.M., "Recent progress in Regge calculus", Nucl. Phys. Proc. Suppl., 57, 73-81, (1997). For a related online version see: R.M. Williams, "Recent progress in Regge calculus", (February, 1997), [Online Los Alamos Archive Preprint]: cited on 19 November 1998, http://xxx.lanl.gov/ $\mathrm{abs} / \mathrm{gr}-\mathrm{qc} / 9702006.3 .13$ 